\title{
Semantic Expansion of Search Queries
}

by

\author{
Andisheh Keikha \\ Masters of Science, K.N.Toosi Univerisity of Technology, 2012 \\ Bachelor of Science, Ferdowsi University of Mashhad, 2009
}

\author{
A thesis \\ presented to Ryerson University \\ in partial fulfillment of the \\ requirements for the degree of \\ Master of Applied Sceince \\ in the Program of \\ Electrical and Computer Engineering
}

Toronto, Ontario, Canada, 2016

(C)Andisheh Keikha 2016 


\section{AUTHOR'S DECLARATION FOR ELECTRONIC SUBMISSION OF A THESIS}

I hereby declare that I am the sole author of this thesis. This is a true copy of the thesis, including any required final revisions, as accepted by my examiners.

I authorize Ryerson University to lend this thesis to other institutions or individuals for the purpose of scholarly research.

I further authorize Ryerson University to reproduce this thesis by photocopying or by other means, in total or in part, at the request of other institutions or individuals for the purpose of scholarly research.

I understand that my dissertation may be made electronically available to the public. 
Semantic Expansion of Search Queries

Master of Applied Sceince 2016

Andisheh Keikha

Electrical and Computer Engineering

Ryerson University

\begin{abstract}
One of the major challenges in Web search pertains to the correct interpretation of users' intent. Query Expansion is one of the well-known approaches for determining the intent of the user by addressing the vocabulary mismatch problem. A limitation of the current query expansion approaches is that the relations between the query words and the expanded words is limited. In this thesis, we capture users' intent through query expansion. We build on earlier work in the area by adopting a pseudo-relevance feedback approach; however, we advance the state of the art by proposing an approach for feature learning within the process of query expansion. In our work, we specifically consider the Wikipedia corpus as the feedback collection space and identify the best features within this context for term selection in two supervised and unsupervised models. We compare our work with state of the art query expansion techniques, the results of which show promising robustness and improved precision.
\end{abstract}




\section{Contents}

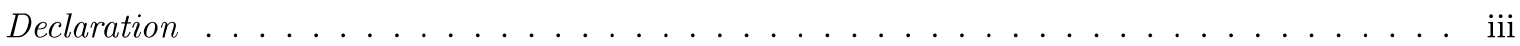

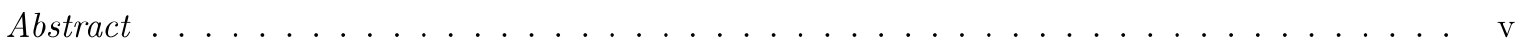

List of Tables . . . . . . . . . . . . . . . . . . . ix

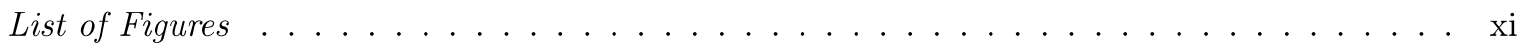

1 Introduction $\quad 1$

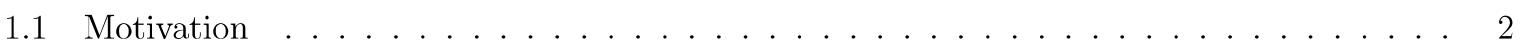

1.2 Problem Statement . . . . . . . . . . . . . . . . . . . . . 5

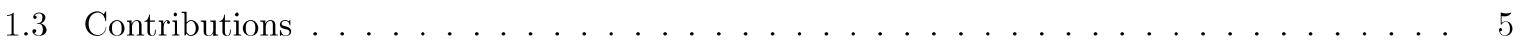

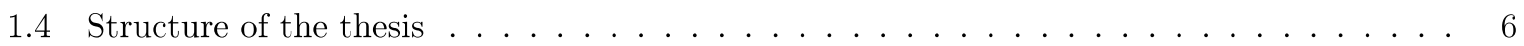

$\begin{array}{lll}2 & \text { Literature Review } & 7\end{array}$

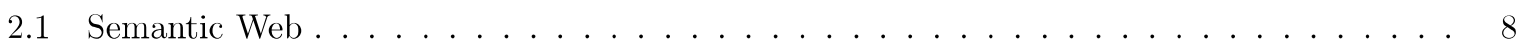

2.2 Search Engine Framework $\ldots \ldots \ldots \ldots \ldots \ldots \ldots$

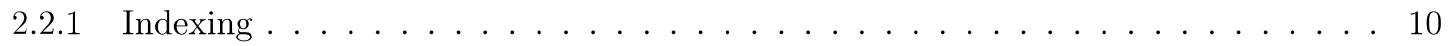

2.2 .2 Query Processing . . . . . . . . . . . . . . . . . . . . . . . 11

2.2 .3 Ranking . . . . . . . . . . . . . . . . . . . . . 16

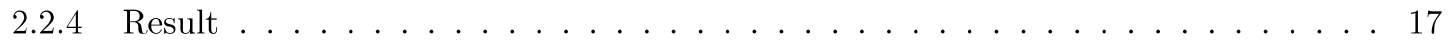

3 The Proposed Approach $r$

3.1 Query Disambiguation and Annotation . . . . . . . . . . . . . . . . . 20

3.1 .1 Unambiguous Queries . . . . . . . . . . . . . . . . 21

3.1 .2 Ambiguous Queries . . . . . . . . . . . . . . . . . . . 22 


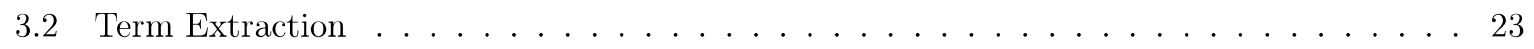

3.2 .1 Unsupervised Term Selection . . . . . . . . . . . . . . . . . 24

3.2 .2 Supervised Term Selection . . . . . . . . . . . . . . . . . . . 27

4 Empirical Evaluations $\quad 30$

4.1 Parameter Tuning . . . . . . . . . . . . . . . . . . . 33

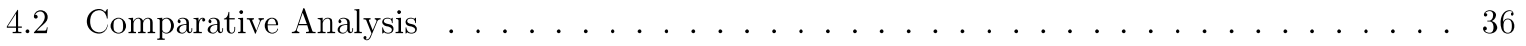

4.2 .1 Unsupervised Term Selection . . . . . . . . . . . . . . . . . . . . 36

$4.2 .2 \quad$ Supervised Term Selection . . . . . . . . . . . . . . . . . . . . . 37

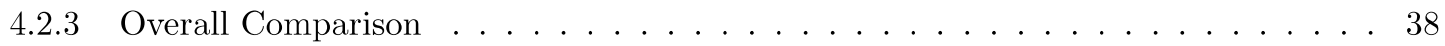

4.3 Robustness . . . . . . . . . . . . . . . . . . . . . . 42

5 Conclusion $\quad 44$

5.1 Future Work . . . . . . . . . . . . . . . . . . . . . . 45

$\begin{array}{ll}\text { References } & 48\end{array}$ 


\section{List of Tables}

3.1 Examples of extracted Wikipedia articles. . . . . . . . . . . . . . . . . 24

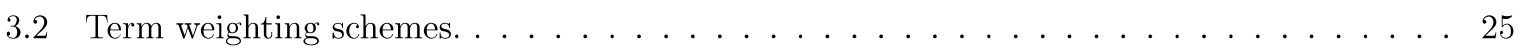

4.1 Results of the unsupervised method $\ldots \ldots \ldots \ldots \ldots \ldots \ldots \ldots$

$4.2 \quad$ Feature Selection Evaluation . . . . . . . . . . . . . . . . . . . 39

4.3 Learning method Evaluation . . . . . . . . . . . . . . . . . . 40

4.4 Comparison on all queries. * determines statistical significance over RMC and RMW

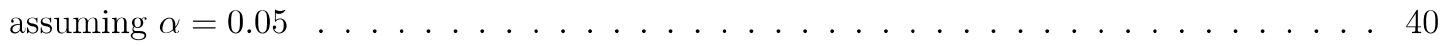

4.5 Examples of feedback documents and their MAP values. . . . . . . . . . . . . . . 41

4.6 Sample queries where Our Unsupervised Approach outperform RMC . . . . . . . . . . . 42

4.7 Sample queries where RMC outperform Our Unsupervised Approach . . . . . . . . . . . 42 


\section{List of Figures}

1.1 User intent VS Query . . . . . . . . . . . . . . . . . . . . 3

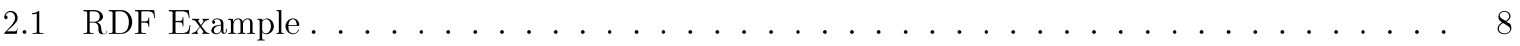

2.2 Simple Indexing . . . . . . . . . . . . . . . . . . . . . . 10

2.3 Indexing with Term Frequency $\ldots \ldots \ldots \ldots \ldots \ldots \ldots$

2.4 Vector Space Model . . . . . . . . . . . . . . . . . . . . . 16

3.1 Approach Overview . . . . . . . . . . . . . . . . . . . . 20

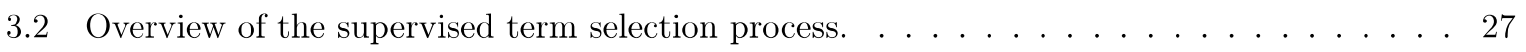

4.1 TREC dataset sample topic and judgment . . . . . . . . . . . . . . . . 31

4.2 Tuning the number of expansion terms: MAP over number of terms. . . . . . . . . . . 33

4.3 Tuning the number of expansion terms: $\mathrm{nDCG}$ over number of terms. . . . . . . . . . 34

4.4 Tuning the number of extracted articles: MAP over number of extracted articles. . . . . . 34

4.5 Tuning the number of extracted articles: nDCG over number of extracted articles. . . . . 35

4.6 Comparative analysis of the robustness results (diagram shows the accumulative values). . 43 


\section{Chapter 1}

\section{Introduction}

Search engines are one of the important areas of information retrieval. Recently, search engines have focused on semantic information from Web documents. The focus on semantics of content has been due to the need to understand and model the intent of the user who submits a search query. There are work in the literature that aim to propose models that retrieve the most related documents to the user's intent based on the query's underlying semantics.

As one of the main aspects of effective search information retrieval, query processing techniques have already received great attention in the literature where different methods such as query expansion, query intent detection, query annotation, among others have been proposed to improve the quality of the search results. In the indexing step, one can improve the results of the search engine by indexing the documents not only based on keywords but also based on semantic information. This semantic information that are indexed can be later used to retrieve the most related Web documents for a given query.

In this thesis, we focus on query processing. Specifically, we focus on query expansion. Query expansion is the solution to the vocabulary mismatch problem in search engines. The vocabulary mismatch problem is when there is a discord between the query words and indexed words. For instance someone searching for the keyword "fish tank" will not get the documents containing "aquarium" in the result set, if no query processing is applied. In query expansion, query words are expanded with some other words that are predicted to be in the related documents to the query. This process can be automatic or interactive. In the automatic approach, the expanded query is submitted to the search engine, instead of the original query and the results are retrieved and shown to the user. In the interactive approach, 
the expanded words are suggested to the user along with the query words, and the user can interactively select the words that she finds relevant.

We propose a novel approach for query expansion that takes into account the vast information available in Wikipedia. The proposed method tries to find the Wikipedia articles related to the query, and then extract the most related words to the user intent as expanded words to the original query that the user submitted. A thorough evaluation has been conducted over the proposed method in comparison to the state of the art, which shows notable improvement.

\subsection{Motivation}

Information Retrieval (IR) can be described as finding documents of unstructured data (natural language) as an answer to a specific information need [35]. In the task of IR there is a corpus available consisting of a collection of documents. This collection can be domain specific or general. A query is the phrase that is conveyed to the system by the user in an attempt to ask for some information, which is mostly represented by keywords in natural language. The purpose of IR is to find all the documents that are relevant to the information need expressed by a query and rank them in the order of relevance. Being relevant means that the user perceives it as containing the information she wants.

The IR system will order the documents based on their likelihood to the query, which means that the documents with higher likelihood must have a priority in the ordering. In a very simple way, the relevance can be interpreted as the existence of the keywords expressed in the query in those documents. This types IR system is the most standard retrieval task which is called ad hoc retrieval [28].

A typical search engine such as Google, is a familiar IR system, in which the document collection is all the web pages that exist on the web. The query is the keyword or the phrase that a user submits to the system to retrieve related information from the web. The user expects to get an ordered list of results in a way that more related results to the user query have a higher priority in the ordering than less related ones.

There are two ways to find the related documents by searching the query keywords in the documents. The first approach is scanning through all the documents to see if they contain the keywords. This approach is called grepping. The second is indexing, in which each keyword is indexed [35]. Some indexes also store the position and the frequency of the word in the document. The decision for the type 


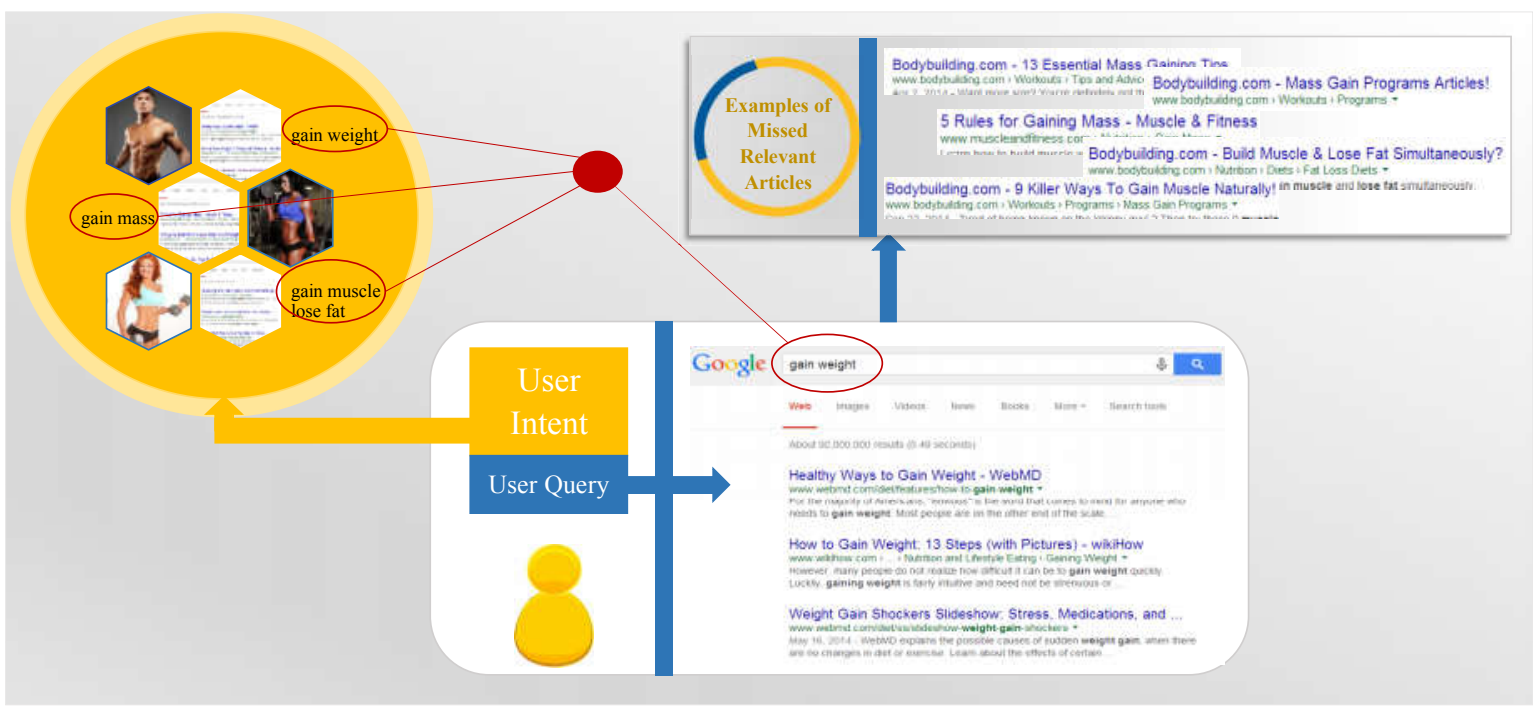

Figure 1.1: User intent VS Query

of information that is saved for each word depends on the retrieval model, the available memory, desired performance, among others. More details on indexing can be found in Section 2.2.1.

One of the challenges in retrieval systems pertains to the correct interpretation of the submitted queries. Since the query is just some keywords in natural language, it can be ambiguous. It has been estimated that $4 \%$ of web queries and $16 \%$ of the most frequent queries are ambiguous. This is mostly due to the length of the query which is normally short [21]. For example someone entering the query "Hotel California" most likely wants to search for Eagles album, however he/she might be interested in hotels in California or a hotel named California.

Other than ambiguity "coverage" is a very important issue. Todays search engines' simple ad-hoc approach retrieves results based on keyword search over indexed web documents with high precision but low recall [21]. In other words, it is very probable that a web document that is much related to a user's intent, but does not contain the words that the user entered in the query does not appear in the results at all. For example looking into Figure 1.1, someone searching for the query "gain weight," wants to find information on how to gain muscle, not gain fat or maybe lose fat. When you enter this query in the 
state-of-the-art search engines like Google, you will get certain results. However when you reformulate it to "gain mass", "gain muscle not fat", "pregnancy gain weight", and "eating disorder gain weight", you will get some completely different results. One might have any of the intents that are mentioned in the reformulations and it would be much better if all of the related results be presented to the user.

Different solutions have been proposed to tackle such problems. One is diversifying search results, so that for an ambiguous query, heterogeneous results are presented. In other words, documents relevant to different aspects of the query will be retrieved in the top results [20]. Search result diversification is defined as presenting documents about each of the aspects of the query in the top results. So that the results are diversified over all of the aspects and different meanings of the query. Another example with a more obvious ambiguity can be the query "java". The intent of the user submitting this query could be "java the programming language" or "java the island". Search result diversification aims to show documents of both meanings in top results.

Search result clustering is another solution to overcome the ambiguity problem, in which the results will be grouped according to different meanings of the query [21]. Therefore, in search systems that use result clustering as a solution to the query ambiguity, all the aspects/meanings of the query is extracted. The interface of the search system is in a way that the user can select from the extracted aspects and see the results of each aspect separately.

Query expansion or reformulation can be another solution to tackle the problem of low coverage and ambiguity, in which the system expands the query to more keywords to cover all of the information that the user may intend. Different reformulations for different aspects of the query can be presented to the user to be selected in an interactive way, or it can be automatically applied within the search engine [11]. For instance, in the "gain weight" query, it was mentioned that different aspects of the query could be: "gain mass", "gain muscle not fat", "pregnancy gain weight", and "eating disorder gain weight". In query expansion in an ad hoc search system, all of these keywords are submitted to the system as the query instead of only submitting "gain weight", and the results of the expanded query is presented to the user. Many researches show that query expansion can improve the results of ad hoc search systems. Different approaches is proposed in query expansion domain to make this expanded set of keywords better in the sense of retrieval. There are also researches on defining different ranking algorithms trying to retrieve the most relevant documents to the users' information need at a higher level.

In order to see how an expansion improves the search result, we need to be able to evaluate it. The 
evaluation of the IR system is based on recall and precision of the system. Recall defines the fraction of the relevant documents retrieved by the system to all of them, and precision indicates the fraction of the retrieved documents to the relevant ones. Different researches may intend to focus on one of these evaluation measures more than the other one based on their goal, while having both high recall and precision is the aim for IR systems.

\subsection{Problem Statement}

In this thesis we are focused on query processing, specifically query expansion. We aimt o improve the search system, proposing a query expansion method that can help the system understand the user's intent. To do so, we need to find related keywords to the query which are not necessarily synonyms of the query keywords. The keywords should be selected with scrutiny and extra care, since wrong inclusion of additional expanded keywords can hurt the results. For example in query "mercy killing", the word "death" might be considered related to the world "killing" while in practice it hurts the results. Some more examples of the words that are bad choices for this query are: "care", "legal", "law", among others.

To explain the problem more formally, assume a query $Q$ consists of $n q$ keywords $q_{1}, q_{2}, \ldots, q_{n q}$. If this query is submitted to a search engine as it is, it will retrieve some results with some accuracy. The accuracy of the results shows how the results are related to the intent of the user. The best result set occurs when the highest ranked results are the most related ones, and there are no unrelated documents in the results. An IR test collection consists of documents, some sample queries, and for each query it has the judgment of that query which shows which document is related and which is not to the query. Based on such judgments, specific metrics have been introduced to evaluate the accuracy of the search system. Knowing the accuracy of the search result for a query based on a metric, we want to improve such accuracy by adding $e q$ related words $W=w_{1}, w_{2}, \ldots, w_{e q}$ to the query $Q$ through an Automatic Query Expansion $(A Q E)$ process and resubmit the new expanded query. The new query is supposed to bring up better set of results according to the user's intent.

\subsection{Contributions}

In this thesis, we provide the following main contributions: 
1. We propose a hybrid approach for the disambiguation of search queries in the context of Wikipedia articles. In our work, we map each query onto a set of coherent Wikipedia articles that collectively represent the underlying semantics of the search query.

2. Given a set of coherent Wikipedia articles for a query, we rank and select a set of terms from those articles for the purpose of query expansion. We employ and empirically compare the performance of various unsupervised schemes for extracting terms from Wikipedia articles.

3. By considering only $20 \%$ of the TREC 2010 dataset for training, and the possible candidate terms for query expansion, we propose a supervised term feature selection function that enables us to select appropriate terms to be included in the query expansion process.

\subsection{Structure of the thesis}

This thesis is written as follow:

1. Chapter 2 - Literature Review: This chapter covers the details of query processing and query expansion techniques.

2. Chapter 3 - The Proposed Approach: This chapter describes the proposed approach of this thesis which is a novel query expansion method using Wikipedia as an external resource and both supervised and unsupervised methods for term selection.

3. Chapter 4 - Empirical Evaluations: In this chapter a thorough evaluation is performed on the proposed approach and the strengths and weaknesses of the approach are discussed.

4. Chapter 5 - Conclusion: In this chapter a summary of the proposed method and the future work of this thesis is presented. 


\section{Chapter 2}

\section{Literature Review}

In this chapter we provide an overview of the Semantic Web, and the concept of Information Retrieval (IR) followed by the different aspects of research in this area. First a simple IR system is described, and then a complete description on different phases in an IR system is presented along with the research challenges in each phase. Also, a more detailed section is dedicated to query processing and specifically to query expansion. 


\subsection{Semantic Web}

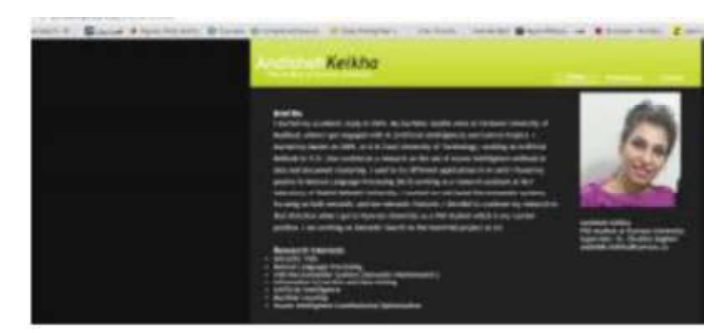

http://ls3.rnet.ryerson.ca/people/keikha/

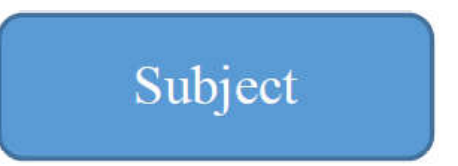

\section{Predicate}

This (url)

This (url)

IsA

Has_FirstName

Person

Andisheh

\section{Statement: This person's first name is Andisheh.}

Figure 2.1: RDF Example

Semantic Web is a systematic change over the traditional web, allowing for the presentation of information in RDF (Resource Description Framework). RDF is a World Wide Web Consortium (W3C) specification that is designed to present metadata/structured data. RDF model represents data as subject-property-object to show the relationship between different resources. Such expressions are called RDF triples. A piece of information is called a statement, and each statement or triple represents a single fact. Figure 2.1 shows an example of a fact. The URL representing the specified person has a first name that is "Andisheh". This is a fact in which the subject is "the person" that is represented by homepage 
URL of the person. The property is "First name", and the object is the literal "Andisheh".

There are a lot of such RDF data already on the web. Linked Open Data (LOD) is the initiative dedicated to producing and linking the so called structured data by building a graph over the existing RDF datasets. DBpedia is one of the largest knowledge bases available on this cloud, that is produced automatically from Wikipedia $[22,49]$.

About half of the knowledge of DBpedia is classified in a cross-domain Ontology, representing classes like Person, Organization or more specific classes like football player, novelist, among others. Different relations are described between the resources in DBpeida, so one can follow and find those properties such as the actor of a movie, or the CEO of an organization [37].

Having such knowledge available, the notion of a more intelligent search has been raised. An intelligent search system is expected to be able to add semantics to the retrieval process [23]. In other words, semantic search is an improved form of search on the web in which the structure and meaning of user queries and web contents are considered through different steps of search. Semantic search systems usually allow more complex queries which can only be processed through knowledge reasoning. Fazzinga and Lukasiewicz have classified semantic search approaches as: 1) those that have a structured query language, 2) those whose query language is non structured which can be a) set of keywords, or b) a natural language statement [23].

\subsection{Search Engine Framework}

A search engine is a practical application of IR. Search engines can be used with small collections, like a limited set of documents or an extremely large collections like the entire web. The collection of documents should be stored in a way that there is a record for each word happening in each document. Saving such records is the task known as indexing. Having all the documents indexed, the search engine receives queries, usually in natural language consisting of some keywords. The query processing phase of the search engine is supposed to process those keywords to extract the semantic of the query and the intent of the user. Not all the search engines have this step. Then the search engine finds the related documents and ranks them based on their relatedness to the user intent, which is called the ranking phase. Finally, it shows the results to the user, in a way that satisfies the user's information needs. The following section describes the details of these steps. 


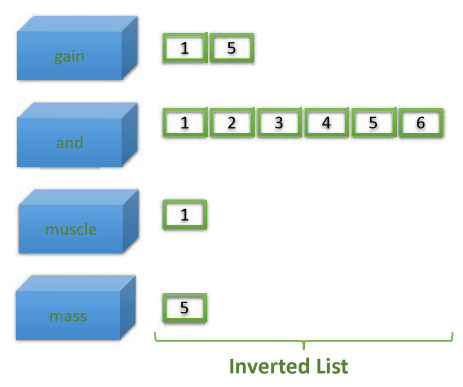

Figure 2.2: Simple Indexing

\subsubsection{Indexing}

Almost all the current search engines use inverted index as the structure of their index. It is called inverted index because in this structure, instead of assuming words as parts of documents, documents are associated with words. The simplest approach for storing inverted index is to save a list of documents ordered by their document number for each word. In other words, if a word has occurred in a document, that document would be in the ordered list of that word, otherwise not. In this simple representation, documents do not have priority over each other for each word, and all are considered as the same. However one document might be much more related to one word than another one. Hence, it is needed to propose a more complicated representation of the inverted index. Therefore, a score is saved for each document associated to each word. This score could be simply the count of the word in the document or more complicated formulas such as tf-idf, BM25, and others. Another information that might be important to save is the position of the word in a document. The list of documents alongside with the score of the word in that document, or any other information that is saved for the index is called the posting list. Figure 2.2 and 2.3 show an example of these kinds of representations. Figure 2.2 shows a simple inverted index without any extra information. Figure 2.3 shows the inverted index plus the count of the word.

Assuming the whole Web as the document collection, storing such considerable amount of data and querying them in real time is something that would need considrable storage space. Therefore, many approaches have been proposed to compress such data such as Delta Encoding, Bit-Aligned Codes, Elias-gama code, among others. 


\subsubsection{Query Processing}

For those queries that are in natural language, the common simple retrieval system is prone to error according to the short length of the query which is normally ambiguous. The most common problem in this domain is the vocabulary mismatch problem which refers to how the user and indexer may use different words (synonymy and polysemy, word inflections) when referring to a concept. For example, an article about gaining weight has the word "muscle" in it, and therefore the indexer only indexes the word "muscle" related to that article. Assuming a user search for "gain mass", although "muscle" and "mass" refer to the same concept, the indexer would not retrieve the document. Such problems may result in an inability of the system to retrieve the desired documents, hence decreasing the recall and precision of the system [11].

To overcome this problem, several solutions have been proposed such as query refinement, pseudo relevance feedback, and result diversification. One of the popular solutions is to expand the query with other words to best capture the actual intent of the user. .

Query processing is not just about query expansion. To better understand the intent of the user two other techniques are available namely: query intent detection, and entity search. In this section we first present a detailed description of various semantic and non-semantic techniques and methods that have been proposed for query expansion. Then an overview of query intent detection and entity search is provided.

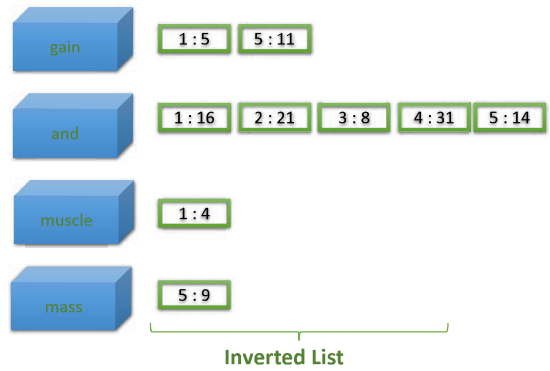

Figure 2.3: Indexing with Term Frequency 


\section{Query Expansion}

Query reformulation and expansion techniques try to tackle the vocabulary mismatch problem, which is primarily concerned with finding semantically similar documents to queries that are not necessarily syntactically similar. Here we review the techniques and approaches that have been proposed to address this problem. Query expansion can be interactive or automatic. In the interactive approach, expansion terms are suggested to the user, and the user can interactively choose or ignore them. In the automatic approach, the words are automatically added to the query without the user noticing. Here we focus on Automatic Query Expansion (AQE). Different approaches used in AQE can be categorized as follow:

- Thesaurus

WordNet [24] is a lexical database for the English language. It groups terms into synonym sets called synsets and presents a definition for each term along with some examples. Other than synonyms, definitions, and examples, WordNet provides hypernyms, hyponyms, meronym, and many other relations between terms. Using this resource for query expansion enables us to use words' synonym, hyponyms, or even some terms from term definitions or glosses as the expansion terms[33]. In other words, instead of only searching for the query terms in the documents, the search engine would search for all the terms that are in the synset or hyponym set or definition of the query terms.

- Relevance Feedback

In Relevance Feedback (RF) methods, relevant documents to the query are considered, and some related words are extracted from those documents for the purpose of query expansion. However in reality, relevant documents are not available, therefore Pseudo Relevance Feedback (PRF) is introduced [11]. PRF takes the initial result set of the specified query and assumes that those results are related to the query. Therefore, it uses them to extract terms related to the query for query expansion.

This approach essentially reinforces the system's original decision, by making the expanded query more similar to the retrieved relevant documents, whereas AQE tries to form a better match with the users' underlying intentions. Although this approach is very promising, it hurts the results in case the initial set of results have non-relevant documents among them [47]. 


\section{- Query Logs}

Query logs contain information about the interaction of the user with the search engine while formulating a query and browsing the results. They are called query logs or click through logs, because they show which documents the user had in fact clicked on after searching for a query [16]. The documents that a user clicks on can be assumed to be related to the intent of the user. The use of click through logs is one of the ways that can be used to find relevant documents and terms to a query [15]. Random walk techniques can be applied on the query-document graph in order to retrieve relevant documents [15]. Also it is possible to extract similar queries from the graph after applying random walk by clustering the queries $[39,18]$.

\section{- Linked Open Data}

Recently researchers have considered the semantic analysis of search queries in the context of the Linked Open Data (LOD) $[18,38,9,14]$. They explore DBpedia, Wikipedia, or Freebase $[5,7]$ to this end. The main difference between such databases and WordNet is that in WordNet, only a limited set of relationships are defined, however in knowledge bases such as DBpedia one can find many different types of relations that are defined as entity properties.

In our work, we have opted to use Wikipedia as the source of information for query expansion. In other researches, it has already been shown that using Wikipedia for query expansion can be more effective than WordNet since Wikipedia is a large, dynamic, and objective knowledge base, which rests on articles that are focused on a single concept $[9,13]$. Also Wikipedia senses cover more search results than WordNet [44]. As such, many recent works are defined around Wikipedia and similar knowledge bases instead of WordNet. Some of the work that use Wikipedia or similar knowledge bases are reviewed in the following.

Bruce et al. [9] extract the aspects of a query using Wikipedia through title matching between Wikipedia articles and query aspects. To find the best aspects, they use a linked probability measure and apply their detected underrepresented aspects in the AbraQ query expansion framework [14]. Similarly, Liu et al [34] represent each aspect of the query as a vector. Query expansion is performed as an iterative method in which in each step a term is added to the expansion set from one of the aspects of the query. Also in their work, aspects can carry different weights. This means that some aspects are more probable to be understood from the query compared to other ones. 
The work in [36] finds the DBpedia concepts related to a unambiguous query. In their first step they extract all the concepts that contain one of the segments of the query in either its label, or in Wikipedia text or text of the link to that Wikipedia article, and in the second step they apply a supervised machine learning method to rank their list of extracted concepts. They evaluate their approach by testing how the extracted concepts are related to the query, hence their approach is not concerned with the term selection part which is one of the important contributions of our work. Moreover, for the training purposes of the thesis, the features are extracted from manually annotated documents.

In another work, [48] proposes a similar idea to our work, which we compare to as the baseline. For entity article selection, they group queries into three classes (EQ: specific entity, AQ: BQ: broad), the first two groups are queries for which a Wikipedia article with the exact same title can be found. For AQ they apply a heuristic disambiguation method and at the end they select one entity for the query to select the terms from. For term selection, they use a parametrized formula to weigh terms and for finding those parameters, they apply a supervised learning method on a training set. The authors only report their results for the EQ and $\mathrm{AQ}$ queries. In our work, we propose a novel method for term extraction from Wikipedia article (which can be more than one) for a query. Also we evaluate the proposed method on all the queries even if a Wikipedia article with the same title cannot be found. For such queries, we propose a method to extract entities related to the query. Such queries are actually the most challenging ones.

Another interesting research is the work of Bendersky et. al [6] which is a relevance model over any unstructured data source. To weight the terms for expansion, they use a parametrized approach, and for parameter tuning they use a supervised learning algorithm over a training set. In our work we specifically use Wikipedia instead of different sources and we believe this choice makes the articles to be more uniform and less prone to error, since our concept extraction is specifically designed for Wikipedia.

\section{Query Intent Detection}

Query intent detection is a solution to the ambiguity of the query. Having search engines work on specific domains or intents makes them become more accurate and easier to adapt, than a general search engine. Therefore, a search framework can consist of search engines for different aims which are called vertical search engines, and the base search engine needs to find out the intent of the user to classify the query to one of the intents and pass the query to the the vertical search engine specific to that domain $[8,4]$. 
For example "download mp3" is a transactional query because it refers to an action via Internet, while "how to download mp3" is an informational query, in which the user wants to get some information on the process of downloading an mp3. Research in this area propose methods to classify a query to one of the predefined classes.

\section{Entity Search}

Entity search is focused on finding information about semantic resources. For example "Alice in wonderland 2010 cast" is a query that asks about the cast of a movie. Assuming Dbpedia as the base knowledge base, the phrase "Alice in wonderland" can be annotated as the movie "Alice in wonderland 2010" that has the page on Dbpedia with uri http://dbpedia.org/page/Alice_in_Wonderland_(2010_film), also the word "cast" can be annotated to the concept http://dbpedia.org/page/Cast on Dbpedia. To this end, many researchers have proposed techniques that are focused on extracting entities from open semantic knowledge bases available on LOD from natural language queries. In other words, a user enters a query just like the one they enter in a typical search engine, and such systems can give them exact answers from structured information available on LOD.

Querying RDF ontologies and semantic web with natural language queries is a recent research area. In this context, the query can be a complete sentence of a "wh" question, or a telegraphic query which is an incomplete question consisting of some keywords [46]. Also their approaches may differ in the sense of their output. Although all of the existing work provide some entities as output, most of them, try to convert the natural language query into a SPARQL query, and then run that query on an RDF knowledge base [46]. As one of the most prominent work in this area we can refer to the work of Sawant and Chakrabarti [45] which focuses on a specific type of entity search queries. Queries that contain some words (selectors) that are meant to occur in the result documents, however some other words in the query phrase are hint words that specify the type of the entity sought by the query. Also it is very important that they are working on telegraphic queries that are typically sent to search engines, which means queries that are not well-formed natural language questions or sentences. They make use of an annotated corpus to calculate the probability of query words be the selector or the hint part of the query and then extract a structured representation for it. 


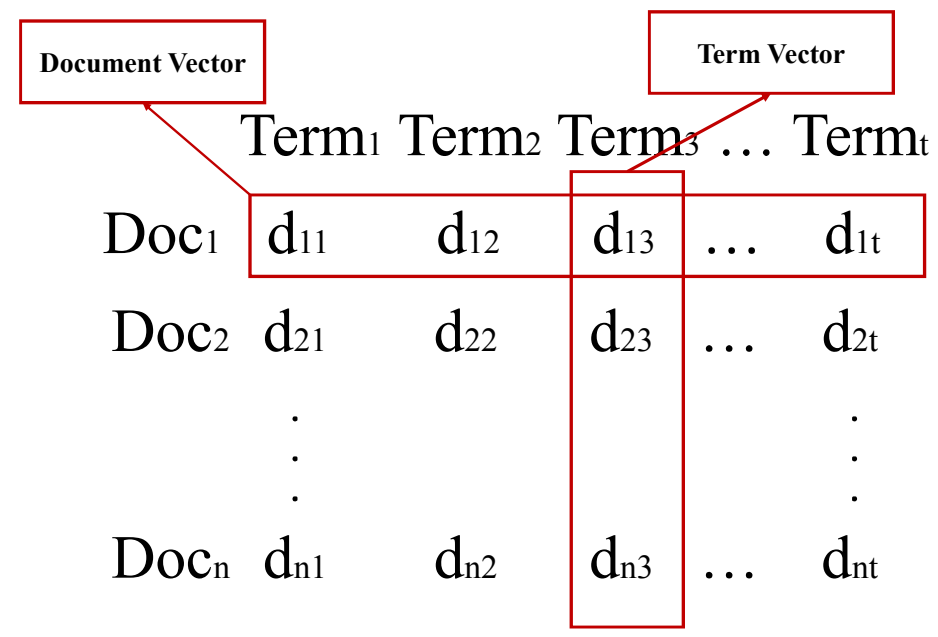

Figure 2.4: Vector Space Model

\subsubsection{Ranking}

Ranking is the core of a search engine. It takes the transformed query from the query processing step as input and generates a ranked list of documents as the result set. The retrieved list of results is supposed to be relevant to the query in a way that high ranked results be more relevant to the intent of the user than low ranked ones. Relevance is a very complex concept and there has been many researches working on this area over the years.

Many different relevance models have been proposed. The most traditional approaches are: Boolean and vector space models. In Boolean Retrieval which is also called exact match retrieval, the documents which exactly match the query specifications are assumed to be relevant, and all others non-relevant. In this model all the documents that are retrieved as relevant are the same in terms of ranking.

In vector space model, as shown in Figure 2.4, each term has a weight vector, where each item of the vector (weights) shows the weight of that term in the corresponding document. Also each document has a vector over terms. Given such vectors, the documents can be ranked given the distance between the vector representing the document and the query. The weights determined for terms can be complicated or simple. For example a simple weight can be the count of the term in that document. Researchers have been working on defining a meaningful and preferably more semantic weight for better results. 


\subsubsection{Result}

The query is ambiguous and can have different meanings. The search engine needs to understand which meaning is the true intent of the user. Also showing the summary of a result web page and different aspects of representation is very important.

Search result clustering and diversification are two approaches that can be effective for overcoming query ambiguity. In Search result clustering, the results are clustered to different groups with definitive labels that describes the aspects (different meanings) of the query. The user then selects which aspect of the query to explore more interactively. One of the very novel researches in this area [21] use "Word Sense Induction (WSI)" approaches for this purpose. Each sense (cluster) of the query is represented as a set of terms, and each search result is represented as its snippet. Therefore, each search result snippet is mapped to one of the clusters related to the query. WSI algorithms are based on some graph partitioning algorithms [21].

Search result diversification is another solution to the problem of ambiguous queries. In fact the aim of this approach is to present different aspects of the results to the user that has searched for an ambiguous phrase. For example someone searching for "java" may have the intent to search for "java programming language" or "java: a type of coffee". It is desirable to show both results as top retrieved results to the user so she can decide for herself which one to chose. However it might be more logical to propose more results and higher ranked results about "java programming language", since it is more probable that this might be the intent of the user. We know this intuitively, while this can be calculated via different frequencies that will be discussed further in this section.

Current approaches in diversification can be categorized into two groups: 1) implicit, and 2) explicit. The implicit approaches consider each document as one topic and try to promote diversification by selecting as much as different and diversified topics as possible and in the meantime cover as much topics as possible. In the explicit approach, different aspects of the query are represented explicitly and again try to cover all and the most diversified set of the aspects in the high ranked results [19]. The former is said to be less effective since the documents' topics might not be related to any aspect of the query.

Furthermore, diversification approaches in higher levels can be classified to two groups of 1) content based, and 2) intent based [3]. Content based diversification focus on reducing redundancy in the 
result documents in a way to prevent letting documents with fewer new information appear in higher ranks. On the other hand, intent based diversification targets the possible ambiguity of the user query. Having all the possible disambiguations of the query, it focuses on presenting all the relevant documents proportionally as higher ranked results. 


\section{Chapter 3}

\section{The Proposed Approach}

The main objective of our approach is to find an accurate representation of the query intent in terms of additional terms that can be effectively used in query expansion. To this end, we use the Wikipedia corpus as the feedback document collection. The primary goals of our work are i) to find a set of Wikipedia articles that can unambiguously represent the underlying semantics of the search query and can be the basis for finding suitable terms for query expansion; and ii) to identify discriminative features that can be used in term selection for query expansion that show improved robustness and performance. Figure 3.1 shows the overview of the steps in our approach.

As shown in Figure 3.1, we first identify a set of candidate Wikipedia articles that can be considered relevant to the query. The extracted articles are evaluated to see whether they are ambiguous or not. We treat ambiguous queries and unambiguous queries differently. Once a set of Wikipedia articles are selected, all the terms in these articles are processed and ranked. For processing the articles to extract terms, we propose two main approaches: unsupervised and supervised term selection. In the unsupervised method, the terms to be included in the expanded query are selected based on the value of a set of predetermined features. In the supervised approach, we first curate a training set, which consists of eight term features. Based on the curated training set, we determine the degree of impact of each feature on the performance and robustness of the query expansion results. To this end, we apply a feature selection method to select the best subset of features, and then employ machine learning techniques to learn the term selection function based on the limited set of selected features. In the supervised term selection method, we select the top terms based on the trained term selection function. 


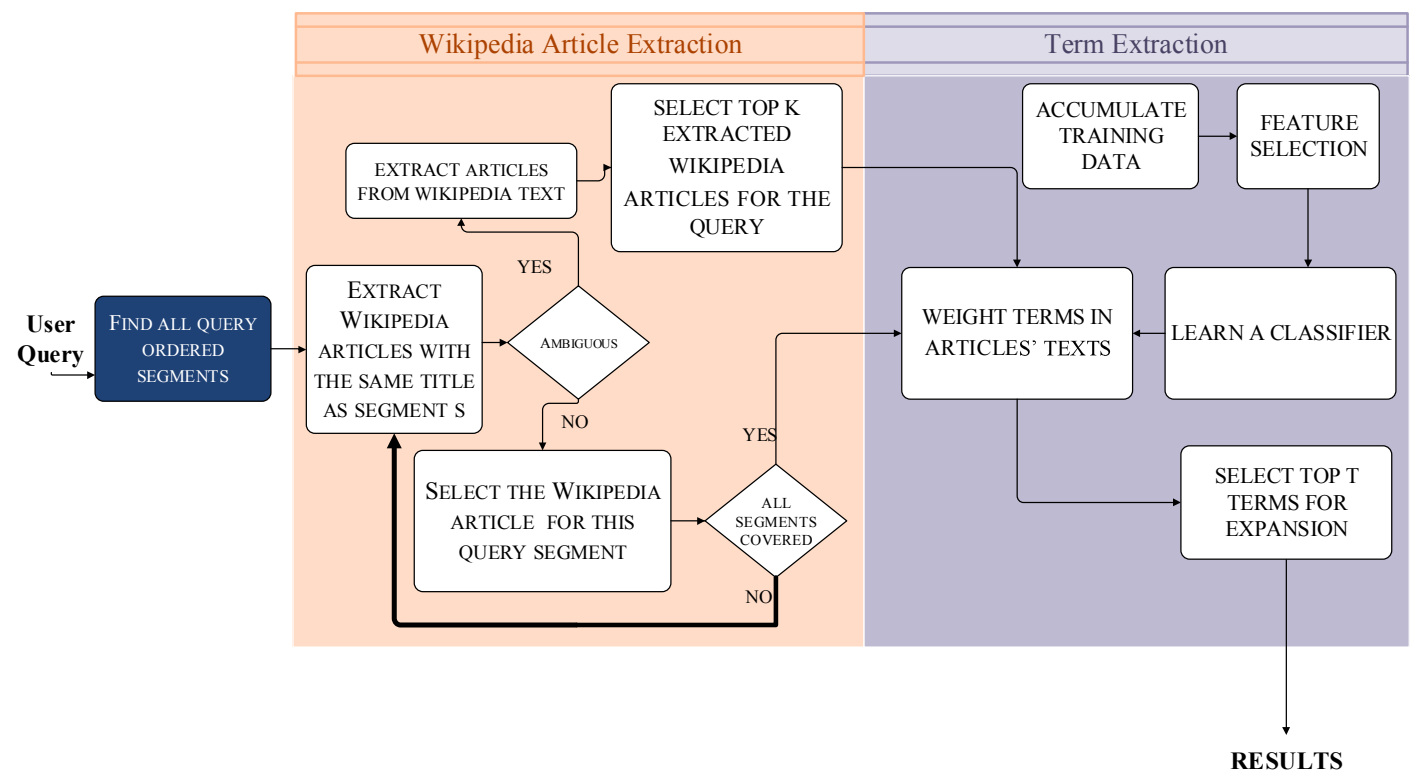

Figure 3.1: Approach overview

We present the details of each step in the following subsections.

\subsection{Query Disambiguation and Annotation}

In order to identify the most relevant Wikipedia articles to a given search query, traditional forms of text annotation $[12,37,25]$ cannot be directly applied due to the very short length of a query and hence, lack of context. Therefore, we consider each query to be a collection of words, which can be used to extract n-grams. We refer to each n-gram extracted from a query as a segment. In the rare case, when a user is looking for one self-contained piece of information and her search query is formulated very accurately, then the largest n-gram in the query, i.e., the query itself, might correspond to one Wikipedia article. For instance, for a search query such as "Barack Obama", one can easily find a corresponding Wikipedia article. However, in reality, users are not necessarily looking for information that have directly corresponding Wikipedia semantics. Furthermore, they might use different syntactic representations to express the same semantic content. Therefore, we need to look into the various segments of the query to 
disambiguate the query and relate it to the most suitable Wikipedia articles. For instance, for the query: "Obama Family Tree", one cannot find a corresponding Wikipedia article; therefore, the semantics of the query needs to be expressed through a combination of Wikipedia articles. For this reason, we look at all the possible query segments, such as "obama family", "family tree", "obama tree", "tree obama", for identifying relevant Wikipedia articles.

In order to identify the most relevant Wikipedia articles for a query, we differentiate between ambiguous and unambiguous queries. We automatically determine whether a query can have multiple senses and therefore be considered to be ambiguous or not. Depending on this, we adopt a different strategy for determining relevant articles and therefore we call this step "hybrid disambiguation". For instance, we can determine that a query such as "Barack Obama" is unambiguous but a query like "Hotel California" is ambiguous.

\subsubsection{Unambiguous Queries}

We first consider all queries to be unambiguous and try to find relevant Wikipedia articles for them. In order to find related Wikipedia articles, we derive all possible query segments as n-grams. We iteratively find the largest n-grams in the query that have a corresponding Wikipedia article. We repeat the process until we have covered all of the terms in the query in at least one of the selected n-grams. For instance, in the query "obama family tree", we first try to identify a Wikipedia article that corresponds to the exact query. Since no such article can be found, we then consider the next possible segments which are "obama family", "family tree" and "obama tree". For the first two segments, articles with the same title are found and as all the terms in the query are covered by these two segments, there is no need to consider the next largest n-grams. Therefore, we represent this query through two Wikipedia articles, namely Obama Family and Family Tree ${ }^{1}$.

While this process finds very accurate Wikipedia article representations for unambiguous queries, it will not be as effective when faced with ambiguous queries. For instance, when applied to a query such as "hotel california", it will not be able to correctly disambiguate between the senses of the query. However, the approach based on the segments allows us to automatically determine whether the query is unambiguous and the extracted Wikipedia articles are reliable or the query is ambiguous and further

${ }^{1}$ Wikipedia articles https://en.wikipedia.org/wiki/Obama_Family and https://en.wikipedia.org/wiki/Family_ tree respectively. 
processing is needed. In order to determine the ambiguity of a query, the list of extracted Wikipedia articles are considered. If any of the extracted Wikipedia articles has a redirection from a Wikipedia disambiguation page, then this shows that the specific query segment that was associated with that article could possibly have different senses. Considering the "hotel california" query as an example, the largest segment would be mapped to the Hotel California article in Wikipedia which is redirected from Hotel_California_(disambiguation) ${ }^{2}$; therefore, pointing to a possible ambiguity in the query. We consider such queries to be ambiguous and further process them as follows.

\subsubsection{Ambiguous Queries}

For the cases where the query is determined to be ambiguous, we adopt a term frequency search of query terms within relevant Wikipedia articles to determine what is the most likely sense of the query. Given search queries are very short and therefore lack proper context, we adopt a popularity-based disambiguation method [31], which assumes that the correct sense of a word, when lacking context, is the one that is the most frequently observed. Therefore, we will assume that the best sense of an ambiguous query is the one that is more frequently observed on Wikipedia. Our ranking strategy allows us to disambiguate search queries based on a popularity-based approach.

To this end, we rank Wikipedia articles based on their relevance to the query terms according to the following equation adopted from [27]:

$$
\operatorname{Rank}_{d}(q, d)=\sum_{t \in q} t f(t, d) \times i d f(t) . \text { lengthNorm }(d)
$$

where $\operatorname{Rank}_{d}(q)$ provides a rank score for document $d$ with respect to query $q, t f(t, d)$ is term frequency of term $\mathrm{t}$ in document $d, i d f(t)$ is the inverse document frequency of the term, and lengthNorm $(d)$ is the normalization value of document text length. TF-IDF is a traditional but very promising approach in ranking the importance of a word. It assumes both frequency and uniqueness of the word at the same time. In equation 3.1, we rank the documents based on the importance of the query terms in those documents. A word is important if it has been repeated many times in one document but not in the others. For example when searching for "DBpedia papers", the word "paper" might not become a significant factor of importance if we search in academic papers, however the word "DBpedia" is certainly

\footnotetext{
${ }^{2}$ https://en.wikipedia.org/wiki/Hotel_California_(disambiguation)
} 
important since it would not be frequently observed in a uniform way in all papers. We also normalize the length of the documents, so that longer documents are not privileged because of their length.

Table 3.1 shows the Wikipedia articles extracted for four sample queries, two non-ambiguous, and two ambiguous, taken from the TREC 2010 dataset. As seen in both of the unambiguous queries, the extracted Wikipedia articles for none of the queries has the same title as the query segments. We have been able to successfully extract the correct Wikipedia article despite vocabulary mismatch because we have considered all redirection links on Wikipedia to denote semantic similarity between the redirected pages. Therefore, if two notions are expressed in different syntactic forms but capture the same semantics, we are able to identify them through the redirects links on Wikipedia. For instance, when searching for the query segment "mercy killing", we were able to determine "Non-voluntary_euthanasia" as the best matching Wikipedia article; therefore, despite the vocabulary mismatch problem, we are able to find the related Wikipedia article to the user query.

Now, for the two ambiguous queries, we first process them as if they were unambiguous; however, in the process if any disambiguation pages are encountered, we note this as an indication of possible ambiguity. For instance, when processing the query "land mine", we encounter Land_mine_(disambiguation $)^{3}$, and we consider the query to be an ambiguous query. Therefore, we employ a popularity based approach for determining the most likely sense of the query. We rank Wikipedia articles based on their relevance to the query and take the top- $k$ to represent the query. We empirically determine the value of $k$ in our experiments.

\subsection{Term Extraction}

Now for a user query, regardless of its ambiguity, we need to identify and select a set of terms that best describe the users' intent; therefore, we consider the Wikipedia articles identified in the previous phase to be the feedback documents within a pseudo-relevance feedback approach and select the top terms from within these documents based on a ranking scheme. We propose two different approaches for this step: 1) unsupervised term selection, and 2) supervised term selection. The details of these two approaches are described in the following subsections.

\footnotetext{
${ }^{3}$ https://en.wikipedia.org/wiki/Land_mine_(disambiguation)
} 
Table 3.1: Examples of extracted Wikipedia articles.

\begin{tabular}{|c|c|c|}
\hline & Query & Identified Wikipedia Articles \\
\hline \multirow{2}{*}{ Non-Ambiguous Queries } & Native American casino & Native_American_gaming \\
\hline & Mercy killing & Non-voluntary_euthanasia \\
\hline \multirow[t]{2}{*}{ Ambiguous Queries } & Land mine & $\begin{array}{l}\text { Land_mine } \\
\text { This_Land_Is_Mine } \\
\text { Land_mine_situation_in_Chech- } \\
\text { nya } \\
\text { Land_mine_situation_in_- } \\
\text { Nagorno-Karabakh } \\
\text { Land_mine_contamination_in_- } \\
\text { Bosnia_and_Herzegovina } \\
\text { Smart_mine } \\
\text { PFM-1 }\end{array}$ \\
\hline & Poliomyelitis and Post Polio & $\begin{array}{l}\text { Poliomyelitis } \\
\text { Post-polio_syndrome } \\
\text { Post-Polio_Health_International } \\
\text { Joseph_Bowler } \\
\text { Post-polio_syndrome } \\
\text { Ivar_Wickman } \\
\text { Ontario_March_of_Dimes }\end{array}$ \\
\hline
\end{tabular}

\subsubsection{Unsupervised Term Selection}

In the unsupervised approach, we exploit eight different term weighting schemes for selecting the most relevant terms to be included in the query expansion process. The terms in the retrieved Wikipedia articles are ranked based on these term weighting schemes and those terms that have the highest value are selected to be included in the query expansion process. These eight weighting schemes are listed and described in Table 3.2. Term Frequency (TF) is a normalized way of calculating the frequency of a term in a given set of documents. In our work and in order to calculate this scheme, all the extracted Wikipedia articles for the query are considered as one document and the TF of each word is calculated. The reason for this is because the different Wikipedia articles that are extracted for a given query are in fact the representatives of the various aspects of the query. Term Frequency-Inverse Document Frequency (TF-IDF) is an extension of the TF scheme which measures how important a word is for a given document within the context of the whole corpus. The IDF scheme offsets frequency when a word is generally very frequent in the corpus. Binary Independence Model (BIM) assumes that words in both the document and query spaces are completely independent (similar to the assumption of the naive 
Table 3.2: Term weighting schemes.

\begin{tabular}{ll}
\hline \hline Function & Formula \\
\hline TF [43] & $0.5+\frac{0.5 \times f(t, d)}{\max \{f(w, d): w \in d\}}$ \\
\hline TF-IDF [40] & $t f(t, d) \times \log \frac{N}{|\{d \in D: t \in d\}|}$ \\
\hline BIM [11] & $\log \frac{p(t \mid R)[1-p(t \mid C)]}{p(t \mid C)[1-p(t \mid R)]}$ \\
\hline Chi-Square [11] & $\frac{(p(t \mid R)-p(t \mid C))^{2}}{p(t \mid C)}$ \\
\hline Weighted Degree $($ WD $)$ & $\sum_{k=1}^{n} W e i g h t\left(n o d e_{i}\right.$, node $\left._{k}\right)$ \\
\hline Weighted PageRank $($ WPR $)$ & Calculated using equation 3.3 \\
\hline WD in Cluster $\left(W D_{c}\right)$ & $\begin{array}{l}\text { WD after WSI graph clustering is ap- } \\
\text { plied }\end{array}$ \\
\hline WPR in Cluster $\left(W P R_{c}\right)$ & $\begin{array}{l}\text { WPR after WSI graph clustering is ap- } \\
\text { plied }\end{array}$ \\
\hline \hline
\end{tabular}

bayes classifier). Furthermore, the Chi-Square scheme works on a similar basis to BIM and measures the importance of a word within the context of the relevant documents. Both of these schemes rely on $p(t \mid R)$ and $p(t \mid C)$, which are the probability of term $\mathrm{t}$ occurring in relevant documents (R) and the probability of term t occurring in the corpus in general (C), respectively as shown in Table 3.2.

Other than the mentioned features, we introduce four additional features that are calculated based on a graph representation of the terms. In order to calculate the graph-based schemes, an undirected graph is constructed over all the terms in the feedback document collection in such a way that the nodes are the terms and the edges are the similarity between the terms calculated though "Resnik Similarity" [41]. Based on this graph structure, we calculate the weighted degree and weighted PageRank value for each node. These two schemes are calculated as shown in equations 3.2 , and 3.3.

$$
W D\left(\text { node }_{i}\right)=\sum_{k=1}^{n} W e i g h t\left(\text { node }_{i}, \operatorname{node}_{k}\right)
$$

where $n$ is the number of nodes that has an edge to node $_{i}$, and Weight $\left(\right.$ node $_{i}$, node $\left._{k}\right)$ is the weight 
of the edge connecting node n $_{i}$ and node $_{j}$.

$$
\begin{aligned}
\text { Weighted }_{P} \text { ageRank }_{\left(\text {node }_{i}\right)=} & \\
& \alpha \times \text { PageRank }\left(\text { node }_{i}\right) \\
+ & (1-\alpha) \sum_{k=1}^{n} \frac{\text { Weight }\left(\text { node }_{i}, \text { node }_{k}\right)}{\sum_{k=1}^{n} \text { Weight }\left(\text { node }_{i}, \text { node }_{k}\right)} \\
& \times \text { PageRank }\left(\text { node }_{k}\right)
\end{aligned}
$$

Equation 3.3 will iterate over all nodes until the PageRank value converges with an error threshold below $\beta$.

These schemes help to extract terms that are more strongly connected in the graph. The nodes with high Weighted Degrees represent those terms that are highly similar to the other terms in the document; therefore, they have a high chance of being central words that could very well represent the topical content of the feedback documents. Furthermore, weighted PageRank shows the probability that a word would be selected based on the connections that it has and its weight with the neighboring nodes. Therefore, a high Weighted PageRank value shows that the term has a high number of strong connections with other terms.

These two schemes are very helpful when the Wikipedia article focuses mainly on one aspect of a concept, however when there are more aspects discussed in one Wikipeia article, there might be some terms that are related to one of the aspects, which might be unrelated to the query. Such terms can be strongly connected to each other, and as a result have a high Weighted Degree and weighted PageRank values, but at the same time harm the results if selected to be included in query expansion. For example for the query "mercy killing", the concept "Non-voluntary_euthanasia" is extracted. In one part of this Wikipedia article, the issue of killing babies being born with a health problem is discussed, and as a result terms like "baby", "child", "parent", and "doctor" are strongly connected, and have high weighted degree, and Weighted PageRank in this context; however, such terms could harm the results if applied in the context of query expansion for the "mercy killing" query.

To enrich our features with some more features that can overcome this problem, we consider using a graph partitioning algorithm that can group the graph into different partitions. We use the Word Sense Induction (WSI) algorithm [21] to partition the graph. Using such algorithms, the graph will be grouped 


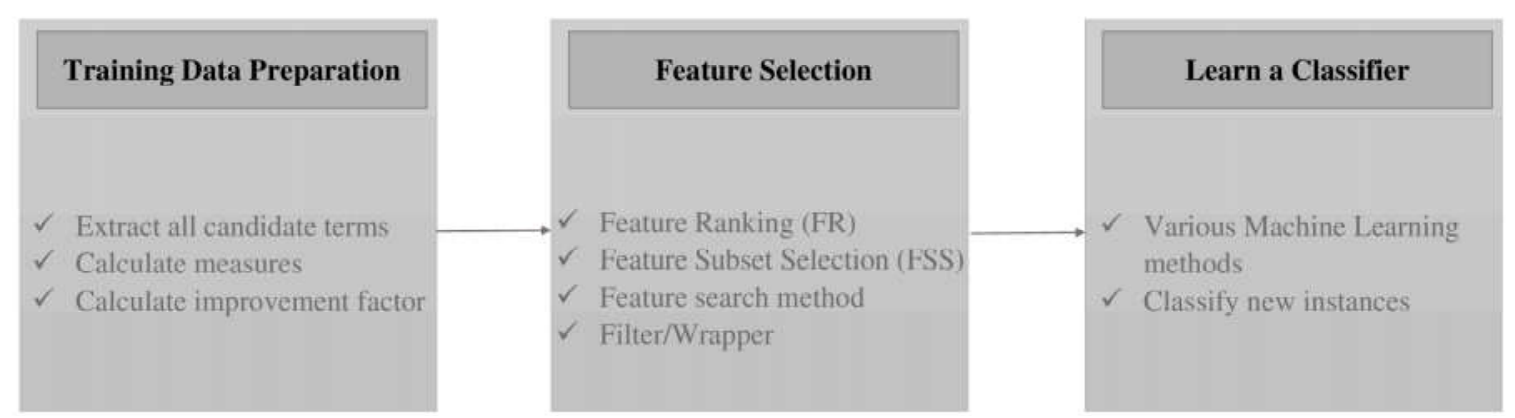

Figure 3.2: Overview of the supervised term selection process.

to strongly connected components in which each component of the graph consists of a set of nodes (terms) that are semantically close to each other. Each component is considered as one semantic aspect of the query, so the terms in each component of the graph are related to one aspect of the query. Applying the algorithm, the graph partitions that the query terms appear in are considered as new subgraphs themselves, and Weighted Degree and weighted PageRank are calculated inside those subgraphs instead of the complete graph. We call these schemes WD in Cluster, and Weighted PageRank in Cluster, respectively. Table 3.2 summarizes the eight schemes used in this step.

\subsubsection{Supervised Term Selection}

Our hypothesis in the supervised term selection method is that there might be a more discriminative combination of the weighting schemes that can more effectively determine better terms for query expansion. For instance, in the unsupervised method, we only consider the weighting schemes separately; however, it is possible that better results would be obtained if these schemes were combined. Hence in the supervised term selection approach, we would like to build a term selection function using a subset of the eight weighting schemes.

To do so, we adopt a machine learning-based method to learn a term weighting function to optimize the effectiveness of query expansion. The overview of the steps of this supervised approach is shown in Figure 3.2. As the first step, we curate a training dataset based on a subset of the queries in our query 
collection (introduced in the evaluation section). The queries are then manually labeled with appropriate Wikipedia articles and best terms to be included in query expansion are determined by an expert. For each of the selected terms, the eight weighting schemes are calculated and used as features. Having in mind that reducing the number of features can defy curse of dimensionality and improve prediction performance [26], we apply a feature selection method to select a subset of the features based on their effectiveness on query expansion. The selected features are then exploited within a machine learning technique to learn an appropriate classifier that would determine whether a term would be included in query expansion or not. The classifier can predict how each candidate term can improve the results of the search engine, and the best terms are selected for query expansion. The details of these steps are described in the following.

Step 1: Training Data Preparation. The training data is manually curated based on queries from the TREC 2010 dataset, for each of the queries of which the terms in the most relevant Wikipedia articles are selected and the values of the eight weighting schemes are calculated. These eight values as well as a label showing how much the selected term would improve the performance of query expansion form the feature space.

In order to prepare the training data, 20 queries were selected from each topic set of the TREC 2010 dataset (totally 60 topics). The queries used in training were not used in the testing process. The candidate terms for all of the queries were extracted and then the query and the expanded query with each term was submitted to a base search engine, i.e. Lucene. The MAP (Mean Average Precision) was calculated for both cases, and the difference between the expanded query and the original query was stored as the degree of improvement. Therefore, a negative value means that the term degrades the result, and a positive one shows improvement. The greater the improvement value is, the more that term contributes to improved results when used for query expansion.

Step 2: Feature Selection. Feature selection can be applied using 1) Feature Ranking (FR), or 2) Feature Subset Selection (FSS) [26]. In the first approach, each feature is evaluated individually, after which they are collectively ranked, and the top $k$ features are selected as the final feature set, while in the latter approach, in each step of the algorithm a subset of features are selected and evaluated. We use the latter approach, since the features are not independent of each other, and the best practice would be not to assume such independence.

An FSS algorithm consists of two steps [1]: 1) finding a subset of features, and 2) evaluating the 
selected subset. For the first step, many strategies have been introduced in the literature such as exhaustive, heuristic and random search [26]. These search methods are often combined with evaluation measures to produce variants for FSS. In our feature selection algorithm, we compare different search methods.

For the evaluation step of FSS, two strategies can be adopted: 1) filter or 2) wrapper. The filter model evaluates features based on a heuristic over the general characteristics of the data and not the schemes that are expected to be learned, while the wrapper will apply a classifier over the data to evaluate the features [42]. Also the wrapper approach evaluates and improves the feature set based on the same scheme that will be optimized by the learner and thus could be more effective than the filter model [26]. The problem with the second approach is its performance on very large datasets, but in our case since our training data includes only 60 samples, the wrapper approach would be quite feasible; hence, we both approaches to see which one fits our method better.

Step 3: Classifier Training. Once the best subset of features is selected, the features that are selected for each term will be considered to represent that term, and the degree of improvement achieved as a result of including that term in query expansion will be considered to be the target label that needs to be predicted. We employ various machine learning methods such as linear regression, multilayer perceptron, pace regression, $\mathrm{RBF}$ networks and additive regression to train a classifier that would produce the degree of improvement for each input term. Each classifier will take as input the term's features and will predict the degree of improvement that is likely to be achieved if this term is included in the query expansion process. Once all the terms are inputted into the classifier, they will be ranked based on the classifier's output and the top $t$ terms are selected for query expansion. 


\section{Chapter 4}

\section{Empirical Evaluations}

Evaluating is a key to propose better search engines, and to find out if an approach is effective in terms of improving a search engine. Other than effectiveness, efficiency and performance can be an important evaluation factor in search engines, however the focus of this thesis is on the effectiveness of a search engine. Effectiveness can be determined as a factor to show the ability of a search engine to find better information. It can be defined for a given query and a specific definition of relevancy. In other words if a set of documents is available that we know for a given query how relevant is each document; there are measures that can be defined to show how effective is one search engine over the selected documents for a given query. The set of relevancy definitions is called judgment, and the set of documents is called test collection which is normally in large scale to be able to evaluate the search engine fairly.

In order to empirically evaluate our work, we used the NIST Special Database 23/NTREC Disk 5 database (the query set and judgments). The contents of the databases are as follows:

1. The Los Angeles Times dataset: This dataset consists of 131,050 documents, which accounts for $475 \mathrm{MB}$ of data. It includes $40 \%$ of the articles published by the Los Angeles Times newspaper in the two year period from Jan 1, 1989 to December 31, 1990.

2. The second dataset is based on the Foreign Broadcast Information Service (FBIS) data. This dataset consists of approximately 130,000 documents which is about $470 \mathrm{MB}$.

Each of the two datasets consists of three collections: i) a set of documents, ii) a set of queries (called topics in TREC) that can be answered by retrieving a subset of the documents, and iii) the expected 


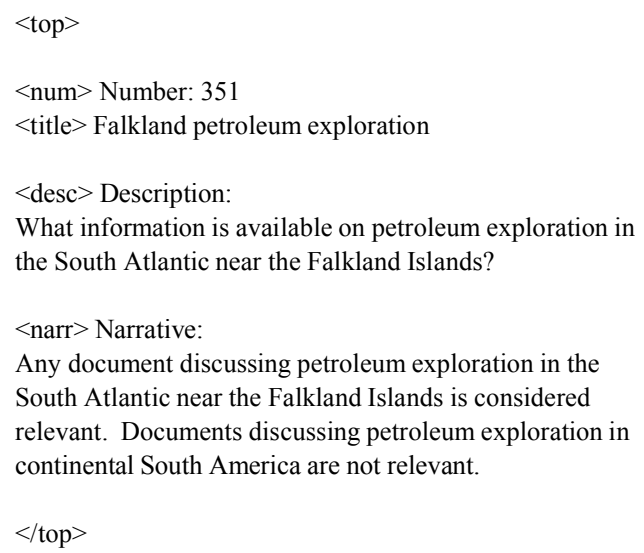

(a)

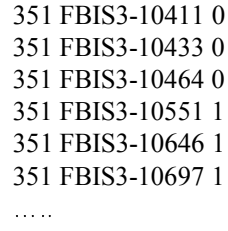

(b)

Figure 4.1: TREC dataset sample topic and judgment

result sets for the queries, known as the relevance judgments. There are only three topic sets that are determined for ad hoc retrieval evaluations: 301-350, 351-400, and 401-450. These are the standard topic sets used for evaluating ad hoc retrieval models.

Each topic (query) in the TREC dataset looks like Figure 4.1-(a), consists of a unique number, title indicating the short query, a description that describes the user intent in a complete question, and a narrative that describes which documents are the expected to be retrieved.

The judgment file as it can be seen in Figure 4.1-(b) consists of the number belonging to the query, an ID that is the document ID constructed from letters, numbers and dash line, and a relevancy number. The higher the relevancy number is the more related the document is to the specified query. For this dataset we only have 0 as non-relevant, and 1 as relevant.

For the purpose of comparative analysis, we compared our work with Relevance Model (RMC), as well as a Relevance Model based on Wikipedia (RMW) as two baselines [48]. These two methods propose state of the art query expansion methods that are vastly used for comparative analysis in this domain $[32,48,17,2]$. For the RMC method, we use the implementation provided through the Indri framework, which is an adaptation of Lavrenko's relevance model [32]. For the R.MW expansion method, we use the Lucene search engine to index and retrieve Wikipedia articles for determining the feedback document set for expansion. We employ two commonly used evaluation measures for evaluating our work, namely: 
i) Mean Average Precision (MAP), and ii) Normalized Discounted Cumulative Gain (nDCG) [30, 10]. MAP considers the performance of the search engine in different recall levels formalized as follows:

$$
\begin{gathered}
\text { Average_} P(q)=\frac{\sum_{k=1}^{n} P(k, q) \times \operatorname{rel}(k, q)}{|R|} \\
M A P=\frac{\sum_{q \in Q} \text { Average_} P(q)}{|Q|}
\end{gathered}
$$

where $P(k, q)$ is the precision at position $\mathrm{k}$ in the result list retrieved for query $q$ and $\operatorname{rel}(k, q)$ is an indicator function equaling 1 if the item at rank $k$ is a relevant document to query $q$, zero otherwise. $Q$ is the set of all queries that are submitted to the search engine, $n$ is the number of documents in the result list and $R$ is the set of relevant documents.

Furthermore, nDCG is a measure that is designed based on two important principles: 1) highly relevant documents are more important than slightly relevant ones, and 2) The higher the rank of a relevant document is, the less desirable it is, because the users are less likely to check them. nDCG is formalized as follows:

$$
\begin{gathered}
D C G_{p}=r e l_{1}+\sum_{i=2}^{p} \frac{r e l_{i}}{\log _{2} i} \\
n D C G_{p}=\frac{D C G_{p}}{I D C G_{p}}
\end{gathered}
$$

where $p$ is the highest position in the list, $r e l_{i}$ indicates 1 or 0 according to its relevance to the query and $I D C G_{p}$ represents the ideal $D C G_{p}$ which means the most relevant documents have the highest ranks.

We also applied a significance test to see if the result of our method is statistically significant compared 


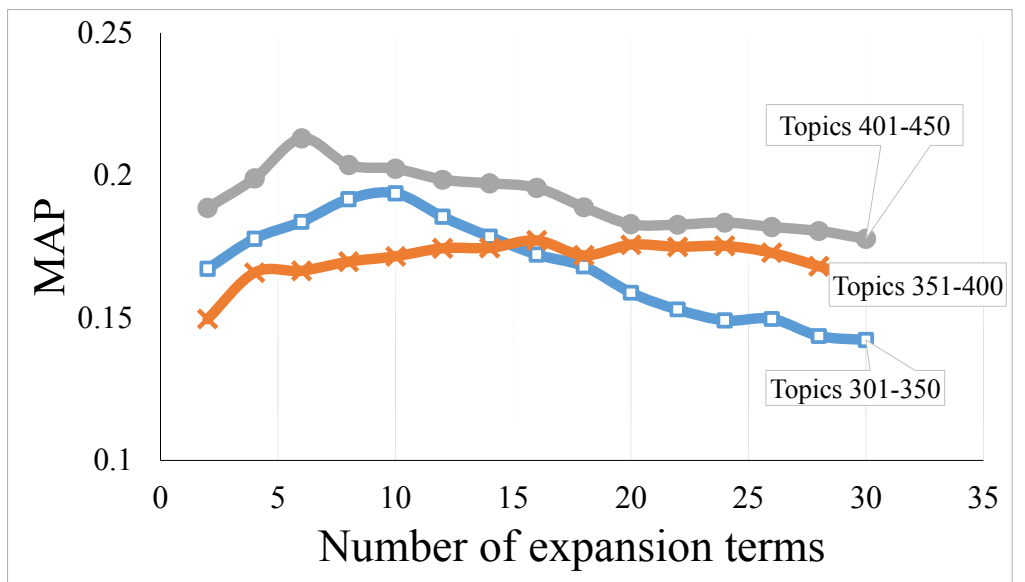

Figure 4.2: Tuning the number of expansion terms: MAP over number of terms.

to other methods. Having the results for methods $A$ and $B$ as a list of numbers (in our case $M A P$ or $n D C G$, The following equation is used to perform the significance test:

$$
t=\frac{\overline{B-A}}{\sigma_{B-A}} \times \sqrt{N}
$$

Where $\overline{B-A}$ is the mean of differences between list of values in $A$ and $B, \sigma_{B-A}$ is the standard deviation of the differences, and $N$ is the size of sample. If this value is less than 0.05 , it shows that algorithm $B$ is more effective than algorithm $A$.

In the following subsections, we first explain how the model parameters are set and then the comparative analysis of our results and the two baselines are provided.

\subsection{Parameter Tuning}

In the first step, we are interested in evaluating the impact of the number of terms used in query expansion on the performance of our work. In other words, we would like to determine the best number of terms to be used in query expansion that would yield the highest performance since including too many terms could negatively impact the results, and too few terms would not address the vocabulary mismatch problem. To this end, we evaluated different number of terms, and the results are shown in Figures 4.2 and 4.3. In this evaluation, we set the size of the feedback document set to seven and used the TF weighting schema to weigh the terms in the feedback document set. Both of these two parameters 


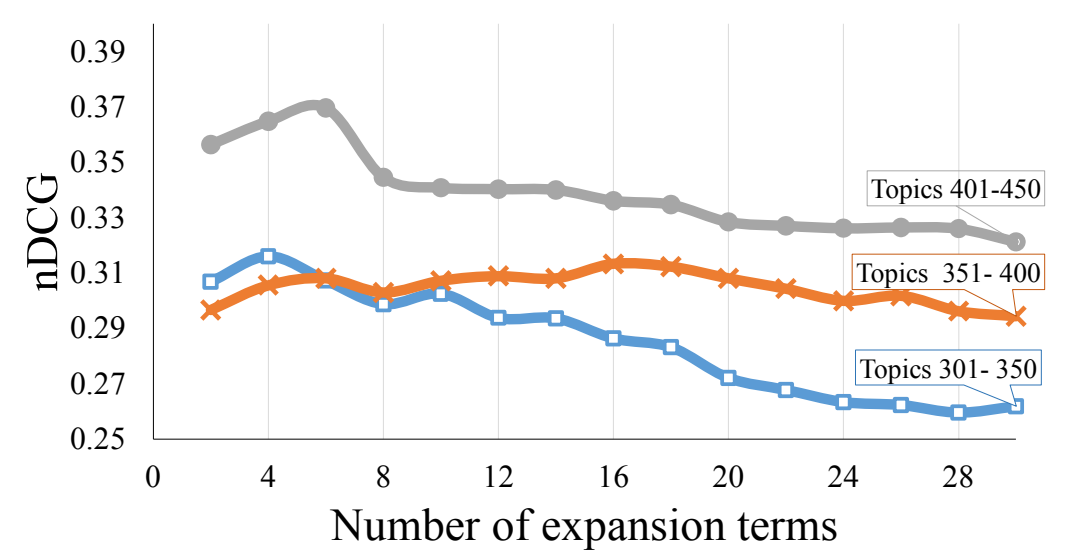

Figure 4.3: Tuning the number of expansion terms: nDCG over number of terms.

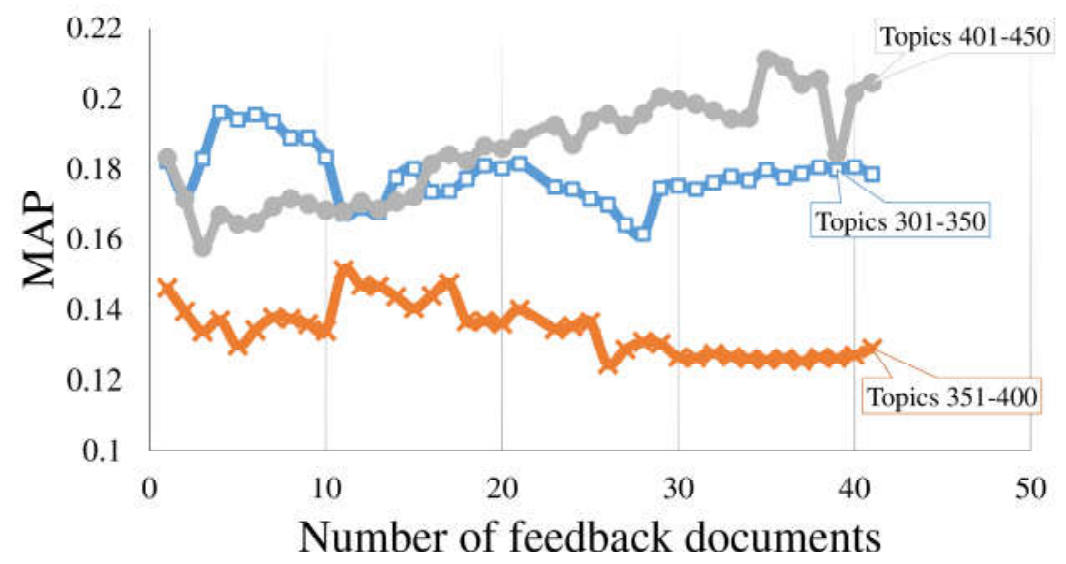

Figure 4.4: Tuning the number of extracted articles: MAP over number of extracted articles. 


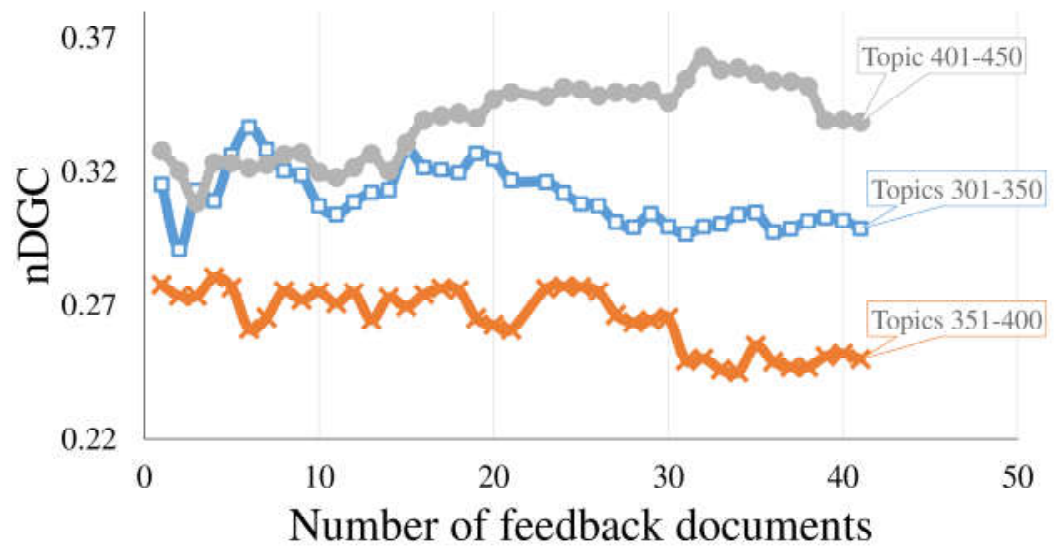

Figure 4.5: Tuning the number of extracted articles: nDCG over number of extracted articles.

will be evaluated in the next steps. Figures 4.2 and 4.3 show the results. In Figure 4.2 , the horizontal axis represents the number of terms, and the vertical axis shows the MAP of the results over all the queries from one of the topic sets. Topics 301-350 are shown in a blue line and topics 401-450 in gray. These two topic sets have almost the same behaviour with the same intensity, while the orange line, which shows topics 351-400, has almost a uniform behaviour. In Figure 4.3, the horizontal axis shows the number of terms and the vertical axis shows the values for nDGC, in which Topics 301-350 and 401-450 have very similar behaviour again and different from topics 351-400. Although topics 351-400 shows almost uniform behavior but it is not totally uniform, with more careful observation, it can be seen that its behavior is similar to the other topics with much less intensity. We can derive from these two figures that very small and very large number of terms for expansion negatively impact the performance of the query expansion method. As shown in Figures 4.2 and 4.3, very large number of terms can demean the results more than a small number of terms. Even though nDCG shows approximately the same behaviour as MAP towards the number of expansion terms, its change is slight, while the change is more recognizable in MAP values. Based on this analysis, it seems reasonable to choose the expansion word set size from the range of 5 to 15 terms.

Furthermore, the size of the feedback document set could potentially impact the performance of the query expansion method. For this purpose, we examined various feedback document sizes and evaluated its impact on MAP and nDCG. Based on the above experiments, we selected the number of expansion terms to be 10 and employed the TF term weighting method in this analysis. Figures 4.4 shows the 
performance for different feedback document sizes. In Figures 4.4 and 4.5 where the horizontal axis shows the number of feedback articles, and the vertical axis shows MAP and nDGC respectively. As seen, the performance of the query expansion method seems to be neither predictable nor impacted by the feedback document size, showing a maximum of $2 \%$ difference on MAP and less than $2 \%$ on nDCG. Therefore, we conclude that the expansion method is not too sensitive to the size of the feedback document set.

\subsection{Comparative Analysis}

Based on the parameter setting analysis in Section 4.1, we compare our work with the baselines introduced earlier and represent a complete analysis comparing: i) Relevance Model (RMC), ii) Relevance Model on Wikipedia (RMW), iii) our proposed unsupervised model, and iv) our proposed supervised model. We perform our experiments on Topics 301-350, 351-400, and 401-450 of the TREC 2010 dataset.

\subsubsection{Unsupervised Term Selection}

As the first step, we evaluate the impact of different term weighting schemes in the unsupervised method on the performance of the query expansion method. Based on the outcomes of the earlier parameter setting studies, we set the number of expansion terms to 10 and the feedback document set size to 15 . The results of the performance of the unsupervised query expansion method based on different term weighting schemes are shown in Table 4.1. In each row of the table, the MAP and nDGC is shown for one of the topic sets. Each column shows one of the schemes. The meaning of this evaluation for each column is that the expansion terms are extracted based on the mentioned schemes in the column topic and then the expansion is evaluated based on MAP and nDGC. In topics 301-350, all of the weighting schemes show reasonable results except TF-IDF, while BIM and Chi-Square show the best improvement among all. In Topics 351-400, BIM and Chi-Square do not perform as expected and the results are not acceptable. In Topics 401-450, all the results are in the reasonable range but still BIM, Chi-square, $W D_{C}$, and $W P R_{C}$ are worse than the others.

Looking into the overall average, it can be seen that TF, TF-IDF, WD and WPR show a slightly better performance over BIM, Chi-square, $W D_{C}$, and $W P R_{C}$. One reason for this could be the fact that in pseudo-relevance feedback each relevant document is expected to be independently relevant to 
Table 4.1: Results of the unsupervised method

\begin{tabular}{c|l|l|l|l|l|l|l|l|l}
\hline \hline Topics & Measure & TF & TF-IDF & BIM & $C h i^{2}$ & WD & WPR & $W D_{C}$ & $W P R_{C}$ \\
\hline \multirow{2}{*}{$301-350$} & MAP & 0.174 & 0.154 & 0.181 & 0.185 & 0.170 & $\mathbf{0 . 1 7 8}$ & 0.178 & 0.165 \\
\cline { 2 - 9 } & nDCG & 0.270 & 0.268 & 0.297 & 0.306 & 0.281 & $\mathbf{0 . 2 9 1}$ & 0.287 & 0.270 \\
\hline \multirow{2}{*}{$351-400$} & MAP & 0.149 & 0.148 & 0.129 & 0.125 & 0.140 & $\mathbf{0 . 1 5 2}$ & 0.148 & 0.141 \\
\cline { 2 - 9 } & nDCG & 0.274 & 0.303 & 0.264 & 0.258 & 0.280 & $\mathbf{0 . 2 8 3}$ & 0.273 & 0.282 \\
\hline \multirow{2}{*}{$401-450$} & MAP & 0.208 & 0.216 & 0.193 & 0.193 & 0.208 & $\mathbf{0 . 2 1 4}$ & 0.194 & 0.197 \\
\cline { 2 - 9 } & nDCG & 0.344 & 0.356 & 0.334 & 0.333 & 0.353 & $\mathbf{0 . 3 6 3}$ & 0.324 & 0.327 \\
\hline \multirow{2}{*}{ Overall Average } & MAP & 0.177 & 0.179 & 0.168 & 0.168 & 0.172 & $\mathbf{0 . 1 8 1}$ & 0.173 & 0.167 \\
\cline { 2 - 9 } & nDCG & 0.296 & 0.309 & 0.298 & 0.299 & 0.304 & $\mathbf{0 . 3 1 1}$ & 0.297 & 0.290 \\
\hline
\end{tabular}

the query, while in our method, each extracted Wikipedia article might not be considered independent of the others. For example, for the query "Alzheimer's Drug Treatment", the extracted Wikipedia articles for the feedback document set are: "Drug_treatment" and "Alzheimer's_disease", each of which covers one aspect of the query, and therefore they are not independent. That could be why the schemes that assume such independency such as BIM, WPR in Cluster, and WD in Cluster do not necessarily result in better performance than those that present an overall measure.

\subsubsection{Supervised Term Selection}

In this section we compare the effect of applying different feature selection approaches and various learning methods on the results of query expansion. Also, we investigate whether the application of feature selection positively affects our results or not. Therefore, as the first comparison, we compare training a fixed classifier method, with and without feature selection. We apply different feature selection approaches and for each of them we show which features are selected. As mentioned in Section 3.2.2, for the feature subset selection methods, we need to select a classifier that evaluates each feature set. For this purpose we adopt the multilayer perceptron as the classifier in all the cases, so that we can only evaluate the effect of feature selection without changing the classifier.

Table 4.2 summarizes the results of using different feature selection methods in combination with the multilayer perceptron. As seen in the table, the best results, highlighted in bold, are observed when either of the following two feature selection methods are employed: i) Genetic Search or Scatter Search, and ii) Latent Semantic Analysis + Ranker. The first feature selection method (i) has selected WD, and BIM as the best set of features for the multilayer perceptron classifier, and the second one (ii) has only selected BIM. The latter feature selection method is a feature ranking method while the former is 
a feature subset selection approach. While the other approaches have occasionally shown comparable results on smaller portions of the topics, these two approaches outperform the other methods on all topics and both evaluation metrics.

In the second set of experiments, we evaluate the impact of the classifier on the results. A consideration that needs to be addressed is that the features selected in the previous stage are the best features based on multilayer perceptron, but we need to apply them on other learning methods. It is important to know that the subset eval feature selection method provides a generic selection of variables, not tuned for/by a given learning machine, which in this case is a multilayer perceptron. Therefore, it is reasonable to use this feature selection method on one predictor as a filter and then train another predictor on the resulting variables as discussed in [26]. As a result, we select the WD, and BIM features that showed promising performance in the previous evaluation as the selected features. The outcome of employing different classifiers is reported in Table 4.3. As seen in the table, the multilayer perceptron achieves the best performance on both of the evaluation metrics and on all three topics. For the multilayer perceptron, the learning rate is set to 0.3 , number of epochs is set to 500 , number of hidden layers is set to 2 , and number of nodes in each hidden layer is set to 3 .

\subsubsection{Overall Comparison}

In this section, we report on the overall comparison of both the supervised and the unsupervised term selection methods compared to the state of the art. Based on the results reported in the previous section, the Weighted Page Rank (WPR) scheme is the better term weighting schemes from among the unsupervised query expansion methods. Furthermore, the subset eval method with Genetic Search and multilayer perceptron as the classifier showed to be the best method among the supervised query expansion techniques. We compare these two methods with the state of the art baseline method, namely Relevance Model on Wikipedia (RMW) [48] and Relevance Model (RMC) expansion [48] methods. Both of the proposed unsupervised and supervised methods perform significantly better across the three topics and on both of the evaluation metrics. This is shown in Table 4.4. The important advantage of the proposed supervised method is that it shows statistically significant improvement over RMC and RMW over all topics and in both metrics.

Our analysis of the observed results based on Relevance Model on Wikipedia (RMW) and Relevance Model (RMC) expansion methods provide some insight as to why both the proposed methods show a 
Table 4.2: Feature Selection Evaluation

\begin{tabular}{|c|c|c|c|c|}
\hline Feature Selection Method & Selected Features & Topics & MAP & $\mathrm{nDCG}$ \\
\hline \multirow{3}{*}{ No feature selection } & \multirow{3}{*}{ All of the 8 schemes } & $301-350$ & 0.164 & 0.271 \\
\hline & & $351-400$ & 0.153 & 0.268 \\
\hline & & $401-450$ & 0.202 & 0.320 \\
\hline \multirow{3}{*}{$\begin{array}{c}\text { Classifiersubseteval }+ \\
\text { (BestFirst Search/Greedy stepwisp } \\
\text { /Linear forward selection) }\end{array}$} & \multirow{3}{*}{ TF-IDF } & $301-350$ & 0.176 & 0.297 \\
\hline & & $351-400$ & 0.158 & 0.287 \\
\hline & & $401-450$ & 0.203 & 0.341 \\
\hline \multirow{3}{*}{$\begin{array}{l}\text { Classifiersubseteval }+ \\
\text { Exhaustive Search }\end{array}$} & \multirow{3}{*}{$\begin{array}{l}\text { WD, tf } \\
\text { BIM, } C h i^{2}\end{array}$} & $301-350$ & 0.182 & 0.290 \\
\hline & & $351-400$ & 0.148 & 0.249 \\
\hline & & $401-450$ & 0.201 & 0.332 \\
\hline \multirow{3}{*}{$\begin{array}{c}\text { Classifiersubseteval }+ \\
\text { (Genetic Search/Scatter Search) }\end{array}$} & \multirow{3}{*}{ WD, BIM } & $301-350$ & 0.194 & 0.312 \\
\hline & & $351-400$ & 0.163 & 0.302 \\
\hline & & $401-450$ & 0.220 & 0.359 \\
\hline \multirow{3}{*}{$\begin{array}{c}\text { Classifiersubseteval }+ \\
\text { Race Search }\end{array}$} & \multirow{3}{*}{$\begin{array}{c}\text { WD, WPR, tf } \\
\text { TF-IDF, BIM, Chi }{ }^{2}\end{array}$} & $301-350$ & 0.178 & 0.297 \\
\hline & & $351-400$ & 0.148 & 0.287 \\
\hline & & $401-450$ & 0.221 & 0.362 \\
\hline \multirow{3}{*}{$\begin{array}{l}\text { Classifiersubseteval }+ \\
\text { Random Search }\end{array}$} & \multirow{3}{*}{$\begin{array}{c}\text { TF-IDF, BIM } \\
C h i^{2}\end{array}$} & $301-350$ & 0.188 & 0.309 \\
\hline & & $351-400$ & 0.158 & 0.297 \\
\hline & & $401-450$ & 0.211 & 0.350 \\
\hline \multirow{3}{*}{$\begin{array}{l}\text { Latent Semantic Analysis } \\
\quad+\text { Ranker }\end{array}$} & \multirow{3}{*}{ BIM } & $301-350$ & 0.188 & 0.306 \\
\hline & & $351-400$ & 0.157 & 0.291 \\
\hline & & $401-450$ & 0.216 & 0.358 \\
\hline \multirow{3}{*}{$\begin{array}{l}\text { Wrapper subset eval + } \\
\text { Genetic Search }\end{array}$} & \multirow{3}{*}{$\begin{array}{l}\text { WPR, } W P R_{c} \\
\text { tf, BIM, } C h i^{2}\end{array}$} & $301-350$ & 0.179 & 0.290 \\
\hline & & $351-400$ & 0.148 & 0.278 \\
\hline & & $401-450$ & 0.201 & 0.349 \\
\hline
\end{tabular}

better performance. In many of the queries, the RMW expansion method ends up including irrelevant Wikipedia articles in the feedback document set for unambiguous queries that degrade the MAP for the query. For example for the query "world bank criticism", the two articles that are extracted in our approach cover the whole intent of the query and do not include any irrelevant Wikipedia articles, while many of the extracted articles in the RMW method are unrelated, although they collectively cover the whole intent. This results in a high recall for the RMW method but at the cost of a lower precision. Table 4.5 shows a comparison of some example queries and the feedback documents for the RMW method and our method. It is important to note that both of our supervised and unsupervised methods use the same set of Wikipedia articles.

We further investigate the performance of RMC compared to our proposed methods. When looking at specific queries where RMC and our approaches have a difference in performance, we were able identify two sets of queries, the first set of which include queries on which our approach performs better than 
Table 4.3: Learning method Evaluation

\begin{tabular}{l|l|l|l}
\hline \hline Learning Method & Topics & MAP & nDCG \\
\hline \multirow{3}{*}{ Linear Regression } & $301-350$ & 0.173 & 0.282 \\
\cline { 2 - 4 } & $351-400$ & 0.160 & 0.289 \\
\cline { 2 - 4 } & $401-450$ & 0.201 & 0.340 \\
\hline \multirow{3}{*}{ Multi layer perceptron } & $301-350$ & 0.188 & 0.311 \\
\cline { 2 - 4 } & $351-400$ & 0.162 & 0.301 \\
\cline { 2 - 4 } & $401-450$ & 0.219 & 0.361 \\
\hline \multirow{3}{*}{ Pace regression } & $301-350$ & 0.181 & 0.288 \\
\cline { 2 - 4 } & $351-400$ & 0.159 & 0.301 \\
\hline \multirow{3}{*}{ RBF Network } & $401-450$ & 0.196 & 0.340 \\
\hline \multirow{3}{*}{ Additive Regression } & $301-350$ & 0.179 & 0.290 \\
\cline { 2 - 4 } & $351-400$ & 0.148 & 0.279 \\
\cline { 2 - 4 } & $401-450$ & 0.203 & 0.331 \\
\hline \hline & $301-350$ & 0.183 & 0.289 \\
\cline { 2 - 4 } & $351-400$ & 0.163 & 0.281 \\
\cline { 2 - 4 } & $401-450$ & 0.220 & 0.359 \\
\hline
\end{tabular}

Table 4.4: Comparison on all queries. * determines statistical significance over RMC and RMW assuming $\alpha=0.05$

\begin{tabular}{c|l|l|l|c|c}
\hline \hline Topics & scheme & RMC & RMW & Unsupervised method & Supervised method \\
\hline \multirow{2}{*}{$301-350$} & MAP & 0.174 & 0.184 & $0.193^{*}$ & $0.194^{*}$ \\
& nDCG & 0.302 & 0.300 & $0.314^{*}$ & $0.313^{*}$ \\
\hline \multirow{2}{*}{$351-400$} & MAP & 0.149 & 0.157 & $0.171^{*}$ & $0.163^{*}$ \\
& nDCG & 0.274 & 0.296 & $0.307^{*}$ & $0.301^{*}$ \\
\hline \multirow{2}{*}{$401-450$} & MAP & 0.208 & 0.206 & 0.215 & $0.222^{*}$ \\
& nDCG & 0.344 & 0.349 & 0.358 & $0.364^{*}$ \\
\hline \hline
\end{tabular}

RMC shown in Table 4.6 and the second set where RMC has a better performance shown in Table 4.7. When looking at the differences between the queries, we notice that the queries where RMC shows a better performance seem to be very specific and on topics that do not necessarily have relevant content on Wikipedia. For instance, for the query "unsolicited faxes" there does not seem to be sufficient relevant articles on Wikipedia to warrant the extraction of a specific feedback document set and therefore the expanded word set is not as accurate in our approach compared to RMC. On the other hand, as shown in Table 4.6, more general queries, such as "airport security" that are on topics that have sufficient coverage on Wikipedia, result in superior feedback document set in our approach and therefore would include more relevant terms in the expansion word set. 
Table 4.5: Examples of feedback documents and their MAP values.

\begin{tabular}{|c|c|c|c|c|}
\hline Query & Feedback documents from RMW & MAP & $\begin{array}{l}\text { Feedback docu- } \\
\text { ments from our } \\
\text { approach }\end{array}$ & MAP \\
\hline $\begin{array}{l}\text { world bank } \\
\text { criticism }\end{array}$ & $\begin{array}{l}\text { Imperial_Bank_of_Persia } \\
\text { Dai-Ichi_Kangyo_Ban } \\
\text { Bangladesh_climate_- } \\
\text { Multi_Donor_Trust_Fund } \\
\text { World_Bank } \\
\text { Bad_bank } \\
\text { Arun_III } \\
\text { World_Bank_Group } \\
\text { Australia_and_New_- } \\
\text { Zealand_Banking_Group } \\
\text { Monetary_reform } \\
\text { Bank_of_America }\end{array}$ & 0.0522 & $\begin{array}{l}\text { World_Bank_Group } \\
\text { Criticism } \\
\text { World_Bank }\end{array}$ & 0.0899 \\
\hline $\begin{array}{l}\text { endangered } \\
\text { species } \\
\text { mammals }\end{array}$ & $\begin{array}{l}\text { United_States_Fish_and_- } \\
\text { Wildlife_Service_list_of_- } \\
\text { endangered_species_of_- } \\
\text { mammals_and_birds } \\
\text { Lists_of_extinct_species } \\
\text { List_of_mammals_of_Aus- } \\
\text { tralia } \\
\text { Fauna_of_Connecticut } \\
\text { Lists_of_animals } \\
\text { Corynorhinus } \\
\text { Longbeaked_echidna } \\
\text { Rhinoceros_(genus) } \\
\text { Fauna_of_Borneo } \\
\text { Canavalia_pubescens }\end{array}$ & 0.0631 & $\begin{array}{l}\text { Endangered_species } \\
\text { Mammal }\end{array}$ & 0.1044 \\
\hline $\begin{array}{l}\text { magnetic } \\
\text { levitation } \\
\text { maglev }\end{array}$ & $\begin{array}{l}\text { Levitation } \\
\text { Bangalore_Monorail } \\
\text { SCMaglev } \\
\text { National_Maglev_Initia- } \\
\text { tive } \\
\text { Electromagnetic_suspen- } \\
\text { sion } \\
\text { Shanghai_Maglev_Train } \\
\text { Inductrack } \\
\text { William_J._Beaty } \\
\text { Maglev }\end{array}$ & 0.4862 & $\begin{array}{l}\text { Magnetic_levitation } \\
\text { Maglev }\end{array}$ & 0.5051 \\
\hline
\end{tabular}


Table 4.6: Sample queries where Our Unsupervised Approach outperform RMC

\begin{tabular}{l|l|l}
\hline \hline Query & $\begin{array}{l}\text { Expansion terms in RMC } \\
\text { not Our Unsupervised Ap- } \\
\text { proach }\end{array}$ & $\begin{array}{l}\text { Expansion terms in Our } \\
\text { Unsupervised Approach } \\
\text { not RMC }\end{array}$ \\
\hline airport security & passeng, thei, aviat & attack, flight, country \\
\hline land mine ban & state, feder, year, million & $\begin{array}{l}\text { action, clearance, interna- } \\
\text { tional, personnel }\end{array}$ \\
\hline pope beatifications & $\begin{array}{l}\text { saint, martyr, pius, rome, } \\
\text { cardinal }\end{array}$ & $\begin{array}{l}\text { church, pope, cathol, } \\
\text { runci, protest }\end{array}$ \\
\hline \hline
\end{tabular}

Table 4.7: Sample queries where RMC outperform Our Unsupervised Approach

\begin{tabular}{l|l|l}
\hline \hline \multirow{2}{*}{ Query } & $\begin{array}{l}\text { Expansion terms in RMC } \\
\text { not Our Unsupervised Ap- } \\
\text { proach }\end{array}$ & $\begin{array}{l}\text { Expansion terms in Our } \\
\text { Unsupervised Approach } \\
\text { not RMC }\end{array}$ \\
\hline Most Dangerous Vehicles & $\begin{array}{l}\text { thei, accid, time, year, } \\
\text { militari }\end{array}$ & $\begin{array}{l}\text { sign, road, image, good, } \\
\text { traffic }\end{array}$ \\
\hline Unsolicited Faxes & $\begin{array}{l}\text { machin, legisl, junk, ad, } \\
\text { bill }\end{array}$ & $\begin{array}{l}\text { call, marketing, direct, } \\
\text { acma, act }\end{array}$ \\
\hline \hline
\end{tabular}

\subsection{Robustness}

A robust query expansion method will improve many and hurt only a small number of queries. The higher the number of improved queries and lower the number of hurt queries are, the more robust the query expansion method is. Robustness is defined as the number of queries that are negatively impacted by the query expansion methods [48]. An ideal query expansion method would improve robustness on any given query. However, in practice, query expansion methods do not necessarily improve the results on all queries; therefore, those methods that improve the results on a higher number of queries are preferred. Figure 4.6 shows the comparison of the robustness for the four methods. For the Supervised approach, the classifier subset eval feature selection with greedy search is applied to select the best features, and multilayer perceptron is used as the learner. In the unsupervised method, WPR is used as the scheme to evaluate the terms. As seen in the Figure, the supervised approach is more robust than the other approaches. The number of queries that their MAP results are improved is significantly higher in the supervised method compared to the other three. The supervised approach makes $68.6 \%$ of the queries better, in comparison to unsupervised method, RMW, and RMC that improve only $54.6 \%, 52 \%$, and $50 \%$ of the queries, respectively. 


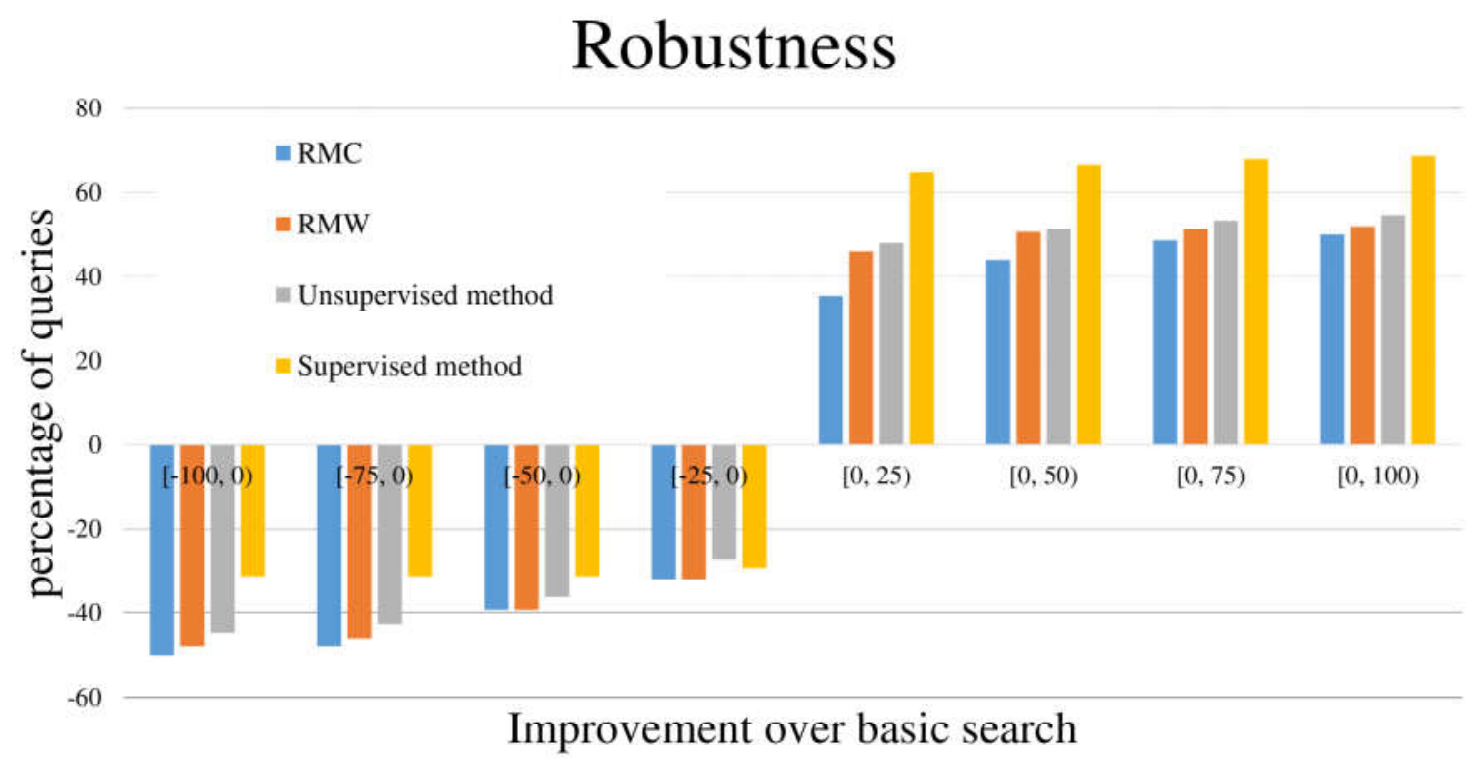

Figure 4.6: Comparative analysis of the robustness results (diagram shows the accumulative values). 


\section{Chapter 5}

\section{Conclusion}

In this thesis we propose two supervised and unsupervised query expansion methods which are inspired by the pseudo relevance feedback query expansion approach considering the extracted Wikipedia articles as feedback documents. Our approaches weigh terms in the specified articles and select the top terms for the purpose of expansion. While in the pseudo-relevance feedback method, there is the possibility that the top results, which are considered to be relevant to the query and helpful for query expansion, contain irrelevant documents that can negatively impact the expansion results; in our approach, we extract Wikipedia articles that are very highly likely to be related to the query and therefore decrease the probability of irrelevant documents being included as a part of the feedback document collection. We make use of the redirect and disambiguation articles of Wikipedia to help overcome the vocabulary mismatch problem. The method proposed for Wikipedia article extraction consider the order of the terms in the query, and searches for the articles with the exact same title as the segments extracted from the query. Then a disambiguation process is proposed for those queries that ends up to disambiguation pages of the Wikipedia. In that case we decided to consider the text of the article for the search process.

Finding the most related articles from Wikipedia, the next step would be determining the best set of terms for query expansion from that articles. In order to do so, we determined 8 different weighting schemes for each term in each article. At this phase, extracting the best set of terms according to the weight values of terms would be the new challenge. We proposed two different approaches, an unsupervised and a supervised approach. In the unsupervised approach, we select the top $t$ terms that have the highest weight. We compared the results of this approach for different weight schemes. For the 
supervised method we used a machine learning approach that can predict the best set of words based on the available schemes. To be able to apply a supervised machine learning method, we need a training set, and therefore we construct one using the TREC dataset. Also we applied a feature selection method to get the best set of features among the predetermined weights. We compared different feature selection and machine learning approaches.

Finally we compared the best results that was obtained from the supervised method that used multi layer perceptron as the machine learning method, with state of the art methods in query expansion. Our results shows significant improvement over traditional approaches.

\section{$5.1 \quad$ Future Work}

Based on the empirical evaluations that we have reported in this thesis, we believe that the proposed approaches (supervised and unsupervised) are most effective when used for expanding information seeking and general queries. As future work, we are interested in exploring the possibility of developing a method that considers non-Wikipedia feedback documents for more specific queries and supervised query expansion proposed in this thesis for generic information seeking queries. This would require the development of schemes that would automatically distinguish between query types.

One other important aspects of discussion in our thesis is the term extraction step. The supervised term extraction produced better results than unsupervised method but not significantly better, however the unsupervised method would be much easier to deploy as it does not require prior training. We are interested in identifying the specific cases where the supervised method is extremely superior to the unsupervised method to determine use cases for each of the approaches.

We used Wikipedia in this thesis as an external resource which showed promising performance compared to using the result set documents as feedback. This means that using a more structured and meaningful resource for terms can help improve the system. While we see that the results are better for more general informative queries than specific ones. Therefore, it would be also interesting to use other sources available on Linked Open Data, and maybe more specific resources for more specific queries. This means that if it would be possible to recognize different types of queries, and their domain to make it specific, such information can help the system. To that aim, we can have vertical search engines, each one specified to a pre-determined domain, and we can have a classifier that determine where each query 
should be submitted. Each search engine can follow the same approach proposed in this thesis, while instead of using Wikipedia for all query types, a domain specific resource can be used. For instance, there is a movie database available on LOD cloud that is a Mixture of content from Wikipedia, Freebase and Geonames ${ }^{1}$. We can distinguish queries that are related to movies, and then use the more specific resource for that. In other words, we can have an intent detection step in our system which classifies the query to a pre-determined class label, and then according to that, a specific resource is used for query expansion.

In the term selection step, Multi Layer Perceptron which is a simple neural network method showed promising results. Another work that can extend our work is to explore other machine learning methods in the term selection step of query expansion. Deep Learning is another promising method that can be a point of interest to be used for the term selection step. Parameter tuning in the neural network approaches and finding the best neural network method with the best set of parameters like the number of hidden layers, and the number of neurons in each layer. would be another step that can be taken to improve the proposed method.

Graph construction for some of the weighting schemes can also be a matter of research. Here we assumed a graph that their nodes are the extracted terms, and the edges the similarity between terms based on Resnik similarity. First we can compare the use of different similarity measures in a subjective matter to see which one gives the best weightings according to human expectation. Second, we can try to take the query terms into account and give each node a weight according to their similarity to the query terms, and then apply a random walk on the graph and the final values of the nodes can be counted as another weight scheme that can be considered in all of the steps of our proposed method. Proposing new ideas for extracting more and more features and weighting schemes can be very helpful in the supervised approach.

Considering the query terms as a factor of importance at the last step of the term selection would be interesting to explore. The relation of each term to the query terms can be another input to the machine learning system, or it can be a new effect on the term selection at the last step. So for example instead of assuming the top $t$ terms in the final extracted sorted list of terms, we can create another ordered list assuming new weights that can be a function of the similarity of each word to the query terms and the output of the classifier. Or even to be more general it can be affected by the similarity of each word to

\footnotetext{
${ }^{1}$ http://datahub.io/dataset/linkedmdb
} 
the query articles as well. 


\section{References}

[1] David W Aha and Richard L Bankert. A comparative evaluation of sequential feature selection algorithms. In Learning from Data, pages 199-206. Springer, 1996.

[2] Bashar Al-Shboul and Sung-Hyon Myaeng. Query phrase expansion using wikipedia in patent class search. In Information Retrieval Technology, pages 115-126. Springer, 2011.

[3] Albert Angel and Nick Koudas. Efficient diversity-aware search. In Proceedings of the 2011 ACM SIGMOD International Conference on Management of data, pages 781-792. ACM, 2011.

[4] Azin Ashkan, Charles LA Clarke, Eugene Agichtein, and Qi Guo. Classifying and characterizing query intent. In Advances in Information Retrieval, pages 578-586. Springer, 2009.

[5] Sören Auer, Christian Bizer, Georgi Kobilarov, Jens Lehmann, Richard Cyganiak, and Zachary Ives. Dbpedia: A nucleus for a web of open data. Springer, 2007.

[6] Michael Bendersky, Donald Metzler, and W Bruce Croft. Effective query formulation with multiple information sources. In Proceedings of the fifth ACM international conference on Web search and data mining, pages 443-452. ACM, 2012.

[7] Kurt Bollacker, Colin Evans, Praveen Paritosh, Tim Sturge, and Jamie Taylor. Freebase: a collaboratively created graph database for structuring human knowledge. In Proceedings of the 2008 ACM SIGMOD international conference on Management of data, pages 1247-1250. ACM, 2008.

[8] David J Brenes, Daniel Gayo-Avello, and Kilian Pérez-González. Survey and evaluation of query intent detection methods. In Proceedings of the 2009 workshop on Web Search Click Data, pages 1-7. ACM, 2009. 
[9] Carson Bruce, Xiaoying Gao, Peter Andreae, and Shahida Jabeen. Query expansion powered by wikipedia hyperlinks. In AI 2012: Advances in Artificial Intelligence, pages 421-432. Springer, 2012.

[10] Chris Buckley and Ellen M Voorhees. Retrieval evaluation with incomplete information. In Proceedings of the 27th annual international ACM SIGIR conference on Research and development in information retrieval, pages 25-32. ACM, 2004.

[11] Claudio Carpineto and Giovanni Romano. A survey of automatic query expansion in information retrieval. ACM Computing Surveys (CSUR), 44(1):1, 2012.

[12] Venkatesan T Chakaravarthy, Himanshu Gupta, Prasan Roy, and Mukesh Mohania. Efficiently linking text documents with relevant structured information. In Proceedings of the 32nd international conference on Very large data bases, pages 667-678. VLDB Endowment, 2006.

[13] Jackie Chi Kit Cheung and Xiao Li. Sequence clustering and labeling for unsupervised query intent discovery. In Proceedings of the fifth ACM international conference on Web search and data mining, pages 383-392. ACM, 2012.

[14] Daniel Wayne Crabtree, Peter Andreae, and Xiaoying Gao. Exploiting underrepresented query aspects for automatic query expansion. In Proceedings of the 13th ACM SIGKDD international conference on Knowledge discovery and data mining, pages 191-200. ACM, 2007.

[15] Nick Craswell and Martin Szummer. Random walks on the click graph. In Proceedings of the 30th annual international ACM SIGIR conference on Research and development in information retrieval, pages 239-246. ACM, 2007.

[16] W Bruce Croft, Donald Metzler, and Trevor Strohman. Search engines: Information retrieval in practice. Addison-Wesley Reading, 2010.

[17] Jeffrey Dalton, Laura Dietz, and James Allan. Entity query feature expansion using knowledge base links. In Proceedings of the 37th international ACM SIGIR conference on Research 85 development in information retrieval, pages 365-374. ACM, 2014.

[18] Van Dang and Bruce W Croft. Query reformulation using anchor text. In Proceedings of the third ACM international conference on Web search and data mining, pages 41-50. ACM, 2010. 
[19] Van Dang and Bruce W Croft. Term level search result diversification. In Proceedings of the 36th international ACM SIGIR conference on Research and development in information retrieval, pages 603-612. ACM, 2013.

[20] Van Dang, Xiaobing Xue, and W Bruce Croft. Inferring query aspects from reformulations using clustering. In Proceedings of the 20th ACM international conference on Information and knowledge management, pages 2117-2120. ACM, 2011.

[21] Antonio Di Marco and Roberto Navigli. Clustering and diversifying web search results with graphbased word sense induction. Computational Linguistics, 39(3):709-754, 2013.

[22] Tommaso Di Noia, Roberto Mirizzi, Vito Claudio Ostuni, and Davide Romito. Exploiting the web of data in model-based recommender systems. In Proceedings of the sixth ACM conference on Recommender systems, pages 253-256. ACM, 2012.

[23] Bettina Fazzinga and Thomas Lukasiewicz. Semantic search on the web. Semantic Web, 1(1):89-96, 2010.

[24] Christiane Fellbaum. WordNet. Wiley Online Library, 1998.

[25] Paolo Ferragina and Ugo Scaiella. Tagme: on-the-fly annotation of short text fragments (by wikipedia entities). In Proceedings of the 19th ACM international conference on Information and knowledge management, pages 1625-1628. ACM, 2010.

[26] Isabelle Guyon and André Elisseeff. An introduction to variable and feature selection. The Journal of Machine Learning Research, 3:1157-1182, 2003.

[27] Erik Hatcher, Otis Gospodnetic, and Michael McCandless. Lucene in action, 2004.

[28] Ben He, Jimmy Xiangji Huang, and Xiaofeng Zhou. Modeling term proximity for probabilistic information retrieval models. Information Sciences, 181(14):3017-3031, 2011.

[29] Anil Jain and Douglas Zongker. Feature selection: Evaluation, application, and small sample performance. Pattern Analysis and Machine Intelligence, IEEE Transactions on, 19(2):153-158, 1997. 
[30] Kalervo Järvelin and Jaana Kekäläinen. Ir evaluation methods for retrieving highly relevant documents. In Proceedings of the 23rd annual international ACM SIGIR conference on Research and development in information retrieval, pages 41-48. ACM, 2000.

[31] Jelena Jovanovic, Ebrahim Bagheri, John Cuzzola, Dragan Gasevic, Zoran Jeremic, and Reza Bashash. Automated semantic tagging of textual content. IT Professional, 16(6):38-46, 2014.

[32] Victor Lavrenko and W Bruce Croft. Relevance based language models. In Proceedings of the 24th annual international ACM SIGIR conference on Research and development in information retrieval, pages 120-127. ACM, 2001.

[33] Shuang Liu, Fang Liu, Clement Yu, and Weiyi Meng. An effective approach to document retrieval via utilizing wordnet and recognizing phrases. In Proceedings of the 27th annual international ACM SIGIR conference on Research and development in information retrieval, pages 266-272. ACM, 2004 .

[34] Xiaohua Liu, Arbi Bouchoucha, Alessandro Sordoni, and Jian-Yun Nie. Compact aspect embedding for diversified query expansions. In Proc. of AAAI, volume 14, pages 115-121, 2014.

[35] Christopher D Manning, Prabhakar Raghavan, and Hinrich Schütze. Introduction to information retrieval, volume 1. Cambridge university press Cambridge, 2008.

[36] Edgar Meij, Marc Bron, Laura Hollink, Bouke Huurnink, and Maarten De Rijke. Learning semantic query suggestions. The Semantic Web-ISWC 2009, pages 424-440, 2009.

[37] Pablo N Mendes, Max Jakob, Andrés García-Silva, and Christian Bizer. Dbpedia spotlight: shedding light on the web of documents. In Proceedings of the 7th International Conference on Semantic Systems, pages 1-8. ACM, 2011.

[38] Greg Pass, Abdur Chowdhury, and Cayley Torgeson. A picture of search. In InfoScale, volume 152, page 1, 2006.

[39] Filip Radlinski, Martin Szummer, and Nick Craswell. Inferring query intent from reformulations and clicks. In Proceedings of the 19th international conference on World wide web, pages 1171-1172. ACM, 2010. 
[40] Juan Ramos. Using tf-idf to determine word relevance in document queries. In Proceedings of the first instructional conference on machine learning, 2003.

[41] Philip Resnik. Using information content to evaluate semantic similarity in a taxonomy. arXiv preprint cmp-lg/9511007, 1995.

[42] Roberto Ruiz, José C Riquelme, and Jesús S Aguilar-Ruiz. Best agglomerative ranked subset for feature selection. In FSDM, pages 148-162, 2008.

[43] Gerard Salton and Chris Buckley. Improving retrieval performance by relevance feedback. Readings in information retrieval, 24(5):355-363, 1997.

[44] Celina Santamaría, Julio Gonzalo, and Javier Artiles. Wikipedia as sense inventory to improve diversity in web search results. In Proceedings of the 48th annual meeting of the association for computational Linguistics, pages 1357-1366. Association for Computational Linguistics, 2010.

[45] Uma Sawant and Soumen Chakrabarti. Learning joint query interpretation and response ranking. In Proceedings of the 22nd international conference on World Wide Web, pages 1099-1110. International World Wide Web Conferences Steering Committee, 2013.

[46] Saeedeh Shekarpour, Edgard Marx, Axel-Cyrille Ngonga Ngomo, and Sören Auer. Sina: Semantic interpretation of user queries for question answering on interlinked data. Web Semantics: Science, Services and Agents on the World Wide Web, 30:39-51, 2015.

[47] Jinxi Xu and W Bruce Croft. Improving the effectiveness of information retrieval with local context analysis. ACM Transactions on Information Systems (TOIS), 18(1):79-112, 2000.

[48] Yang Xu, Gareth JF Jones, and Bin Wang. Query dependent pseudo-relevance feedback based on wikipedia. In Proceedings of the 32nd international ACM SIGIR conference on Research and development in information retrieval, pages 59-66. ACM, 2009.

[49] Liyang Yu. A developer's guide to the semantic Web. Springer, 2011. 